\title{
Comparison of Electron Cloud Simulation and Experiments in the High-Current Experiment
}

\author{
R. H. Cohen, ${ }^{*}$ A. Friedman, M. Kireeff Covo, S. M. Lund, and A. W. Molvik \\ Lawrence Livermore National Laboratory, Livermore CA 94550 \\ F. M. Bieniosek, P. A. Seidl, and J.-L. Vay \\ Lawrence Berkeley National Laboratory, Berkeley, CA 94720 \\ J. Verboncoeur \\ University of California, Berkeley, CA \\ P. Stoltz and S. Veitzer \\ Tech-X Corporation, Boulder CO
}

(Dated: October 7, 2004)

\begin{abstract}
A set of experiments has been performed on the High-Current Experiment (HCX) facility at LBNL, in which the ion beam is allowed to collide with an end plate and thereby induce a copious supply of desorbed electrons. Through the use of combinations of biased and grounded electrodes positioned in between and downstream of the quadrupole magnets, the flow of electrons upstream into the magnets can be turned on or off. Properties of the resultant ion beam are measured under each condition. The experiment is modeled via a full three-dimensional, two species (electron and ion) particle simulation, as well as via reduced simulations (ions with appropriately chosen model electron cloud distributions, and a high-resolution simulation of the region adjacent to the end plate). The three-dimensional simulations are the first of their kind and the first to make use of a timestep-acceleration scheme that allows the electrons to be advanced with a timestep that is not small compared to the highest electron cyclotron period. The simulations reproduce qualitative aspects of the experiments, illustrate some unanticipated physical effects, and serve as an important demonstration of a developing simulation capability.
\end{abstract}

${ }^{*}$ Electronic address: rcohen@llnl.gov 


\section{INTRODUCTION}

As was noted in a previous milestone report [1], stray electrons are becoming increasingly recognized as a factor that must be accounted for in heavy-ion fusion (HIF) accelerators, such that a quantitative understanding is essential. Electron clouds have already been recognized as an issue for accelerator applications such as high-energy physics and spallation neutron sources [2]. The common thread in these contexts is that the electron cloud is an uncontrolled source of negative charge that can alter the ion beam dynamics, possibly leading to increased beam emittance and halo generation, and also potentially driving electron-ion instabilities. For an application such as heavy-ion fusion where there is an economic imperative to have the ion beam fill as much of the cross section of the beam line as possible, electron cloud effects can be a significant factor in limiting the beam pipe fill factor.

The Heavy-Ion Fusion Virtual National Laboratory (HIF-VNL) has been developing both experimental and simulation capabilities in this area, and these capabilities have advanced to the point where a first comparison of simulation and experiment is now possible. This report describes an experiment on the High-Current Experiment (HCX) facility, which is explicitly designed to produce large electron-physics effects, and our efforts to model it. It also summarizes the advances in simulation capability that have brought us to this point.

The remainder of this report is organized as follows: Section 2 describes the experimental setup and results. Section 3 summarizes the advances in electron-physics simulation capability that have occurred over the past year. Section 4 describes our first 3-dimensional simulation of the experiment, which is also our first simulation that self-consistently couples electron and ion physics in a magnetic-focusing accelerator. The simulation results have some points of contact with the experimental results but disagree in other aspects, which we attribute to deficiencies in the employed simulation model. To address these deficiencies,

we have undertaken subsidiary calculations with reduced models, which are described in Section 5: ion slice simulations with specified electron clouds that approximate the electron distribution we expect when we have runs with improved physics, and high-resolution simulations of the end region to elucidate a suitable electron injection model. 


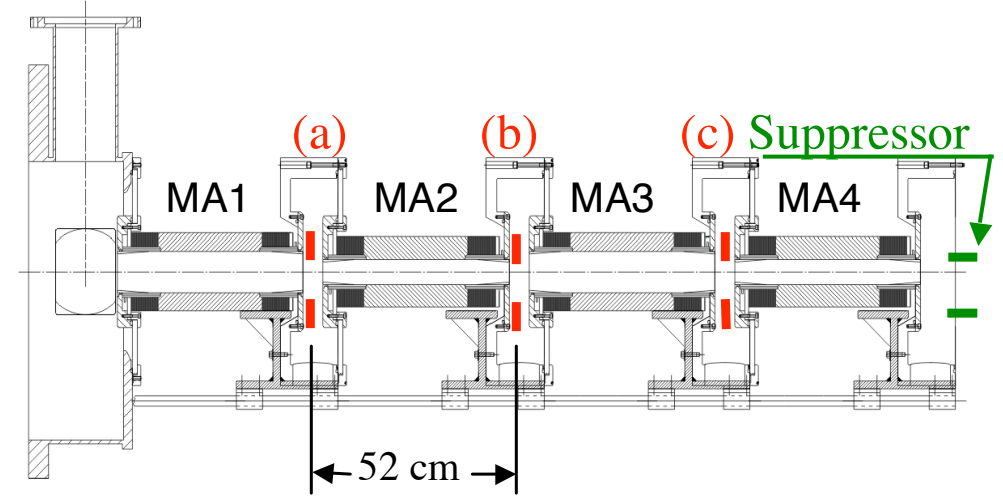

FIG. 1: Electron studies are performed in 4 quadrupole magnets (MA1-MA4) with elliptical bores on the HCX. A suppressor electrode is shown at the right in green, the red electrodes (a) - (c) are clearing electrodes in the drift regions between quadrupole magnets. Every other magnet is rotated $90^{\circ}$, as indicated by the alternating 6 and $10 \mathrm{~cm}$ diameters. A diagnostics region is to the left of MA1.

\section{EXPERIMENT}

Electron studies are performed in four magnetic quadrupole magnets (MA1-4) on the High Current Experiment (HCX) facility, as shown in Fig. 1. These magnets follow 10 HCX electrostatic quadrupoles through which the beam was transported with little or no degradation [3].

During our first tests of diagnostics for measuring electrons in the $1 \mathrm{MeV}, 174 \mathrm{~mA} \mathrm{~K}+$ ion beam in HCX, we found evidence of anomalies in the beam transport through the 4 quadrupole magnets. An example is shown in Fig. 2, wherein the beam was passed through the Phase-1 HCX electron diagnostics $(5.4 \mathrm{~cm}$ diameter, $48 \mathrm{~cm}$ long tubes in MA3 and MA4), before the installation of suppressor and clearing electrodes. This picture shows a single beam pulse, directed through a vertical slit onto the optical imaging diagnostic (a single image from a horizontal slit scan). The complete horizontal scan of the slit shows a Z-shaped $\mathrm{X}-\mathrm{X}^{\prime}$ phase space distribution. ( $\mathrm{X}^{\prime}$ is shorthand for $\mathrm{dX} / \mathrm{dx}$, and represents the transverse angle of an ion's motion relative to the beam axis. The spread of $\mathrm{X}^{\prime}$ values is related to the transverse beam temperature, which ultimately limits the achievable finalfocus spot size.)

Vertical scans of a horizontal slit did not show the Z-shaped phase space, and each beam 


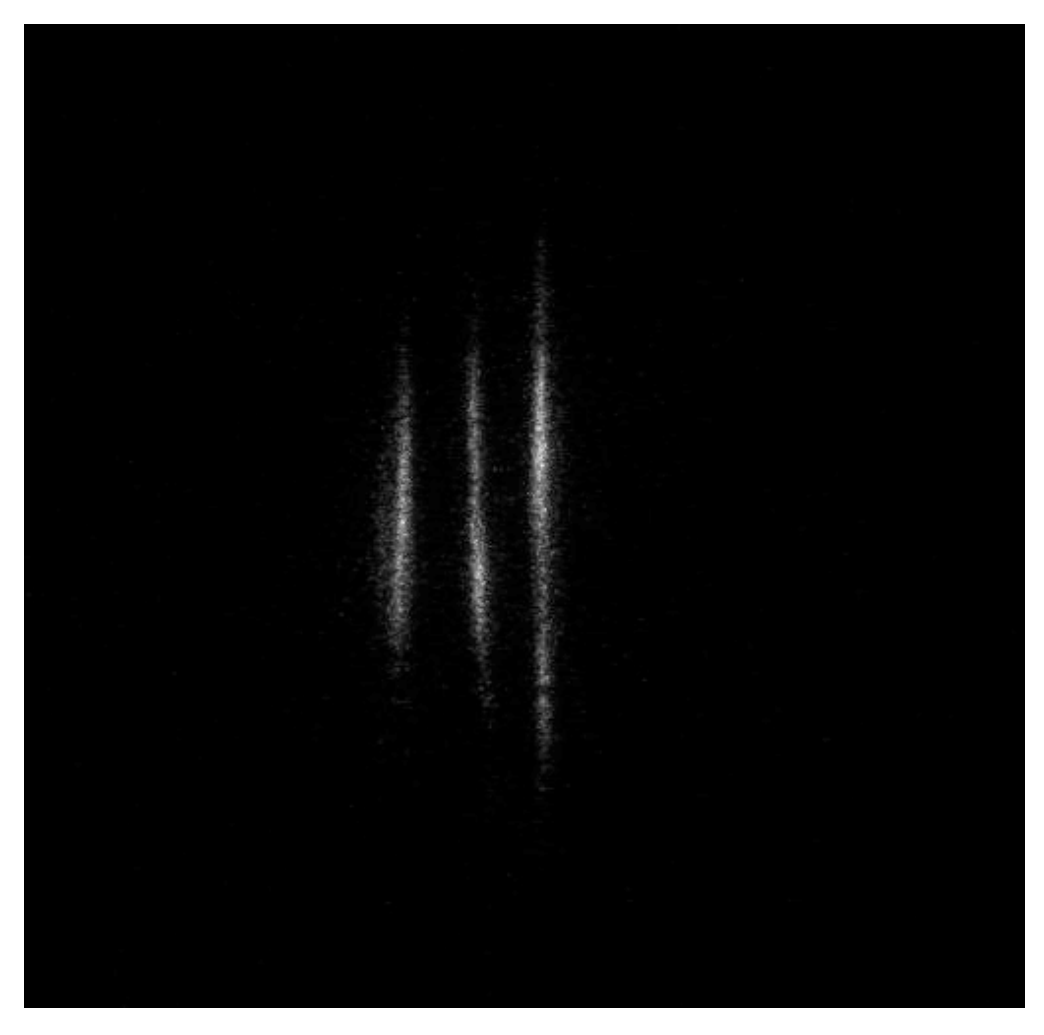

FIG. 2: Image of a single beam pulse that has been directed through a vertical slit. The three stripes indicate that the portion of the beam that passed through the slit had three separate velocity components. This beam had passed through $5.4 \mathrm{~cm}$ diameter cylinders, without electron suppression at the end wall. (Shot 30721039)

pulse showed a single line.

Removal of the Phase-1 diagnostics opened up the aperture to an ellipse of $6 \times 10 \mathrm{~cm}$ principal axes. This changed the beam distortions, but did not eliminate the splitting of velocity space into 3 components, Fig. 3.

Realizing that there was likely to be electron emission from the slit plate during beam impingement, and that this emission might be the cause of the beam trifurcation, we installed a suppressor ring between the final magnet MA4 and the slit plate. The expectation was based on our measurements of electron emission (and gas desorption) from $1 \mathrm{MeV} \mathrm{K}+$ ions impinging upon a stainless plate near grazing incidence [5]. Extrapolating those results to normal incidence yielded an electron emission coefficient of 6 (this coefficent agrees with theoretical estimates [4], although other HIF experiments have suggested that electron emission coefficients can be as high as 10-30) 


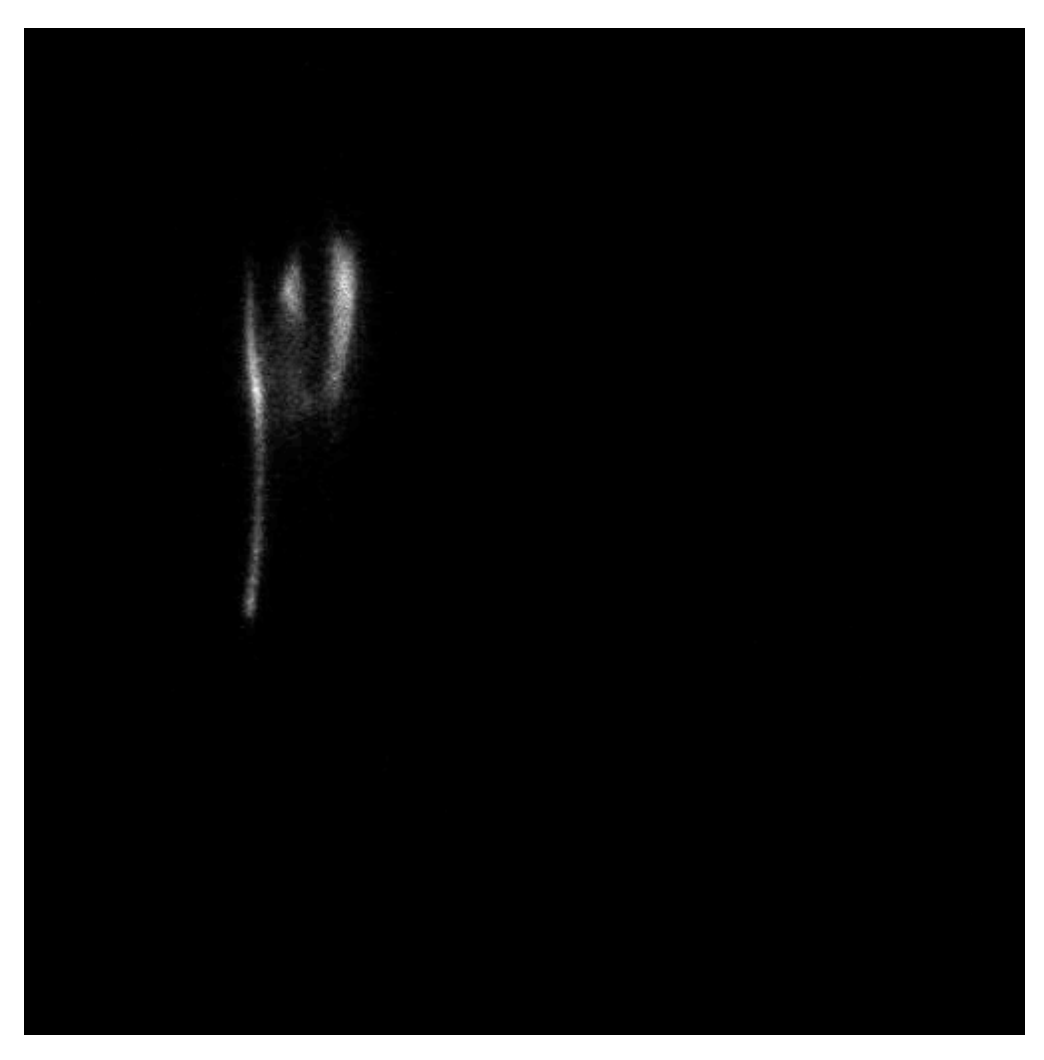

FIG. 3: Cylindrical tubes removed, leaving $6 \times 10 \mathrm{~cm}$ diameter elliptical magnet bore, without electron suppression at end wall. (Shot 31104124)

Operation with the suppressor ring biased to $-10 \mathrm{kV}$ eliminated the phase space distortion, yielding a single image of the slit; see Fig. 4. This demonstrates that trifurcation of the ion beam is caused by electrons emitted off the end wall (in this case, the slit plate). Such electrons might fill the field-free drift region between the slit plate and the end of the last quadrupole magnet. Some of the electrons could drift upstream through the magnets.

We recently repeated these runs in one day, with magnetic quad diagnostics still removed, and testing the effects of varying combinations of the suppressor and clearing electrodes. With all the electrodes biased, $V_{s}=-10 \mathrm{kV}, V_{c a}=V_{c b}=V_{c c}=+9 \mathrm{kV}$, we saw a single image of a slit, Fig. 5. Leaving the suppressor on, but turning off the clearing electrodes gave a similar result, shown in Fig. 6. Leaving the suppression and all clearing electrodes off gave the result shown in Fig. 7, which is similar to that shown in Figs. 2 and 3.

Utilizing all shots from a scan, one can reconstruct the full $x-x^{\prime}$ phase space; an example, with the suppression off, and only the first clearing electrode (a) of Fig. 1 biased (at +9 $\mathrm{kV}$ ), is shown in Fig. 8. 


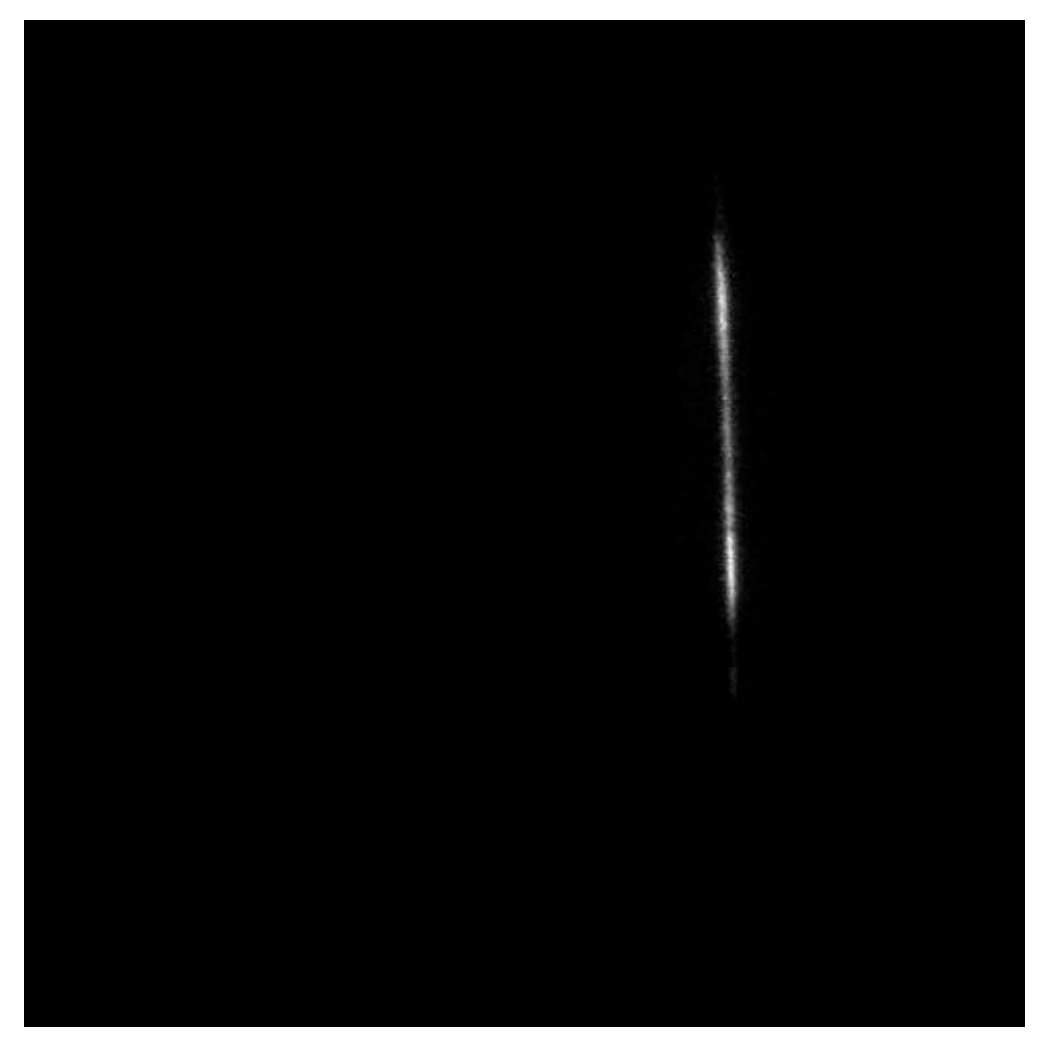

FIG. 4: With electron suppression at end wall. (Shot 31204033)

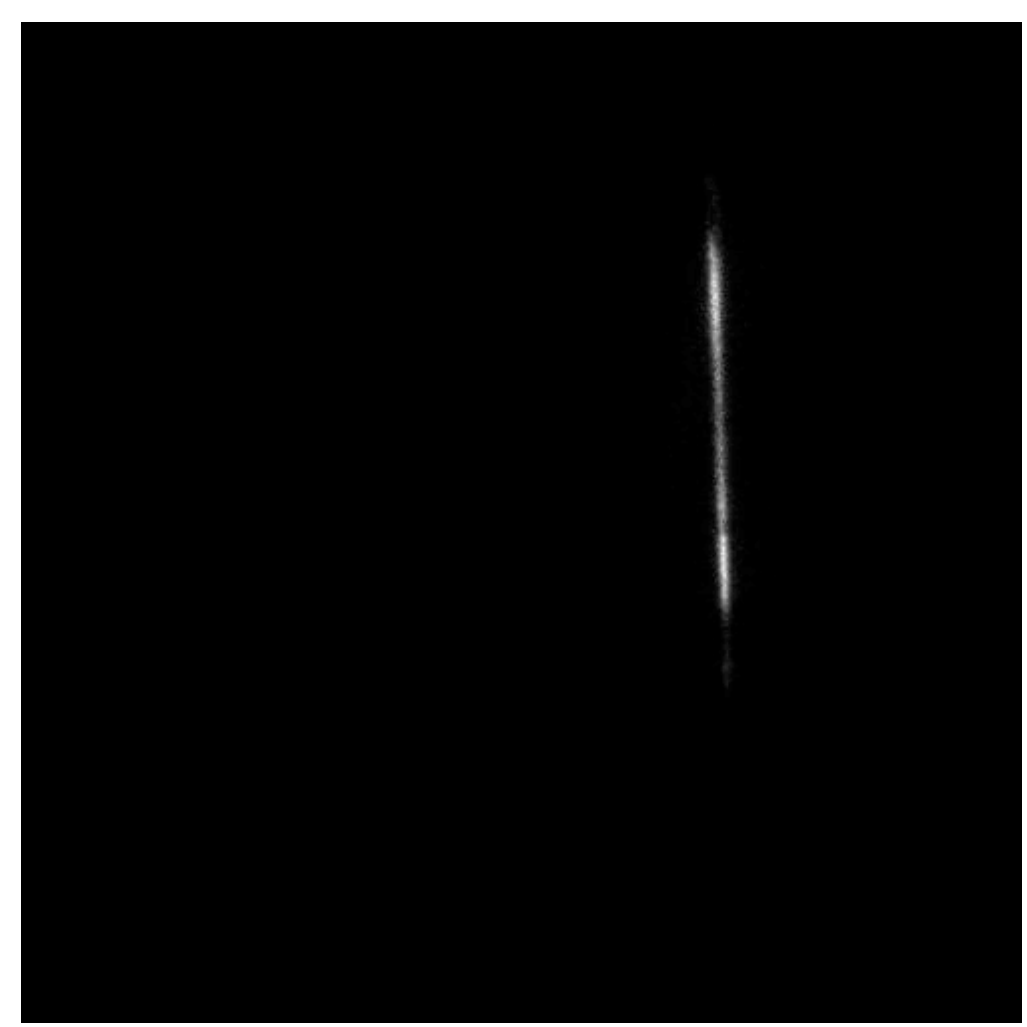

FIG. 5: With electron suppression on and all clearing electrodes biased. (File 409220042). 


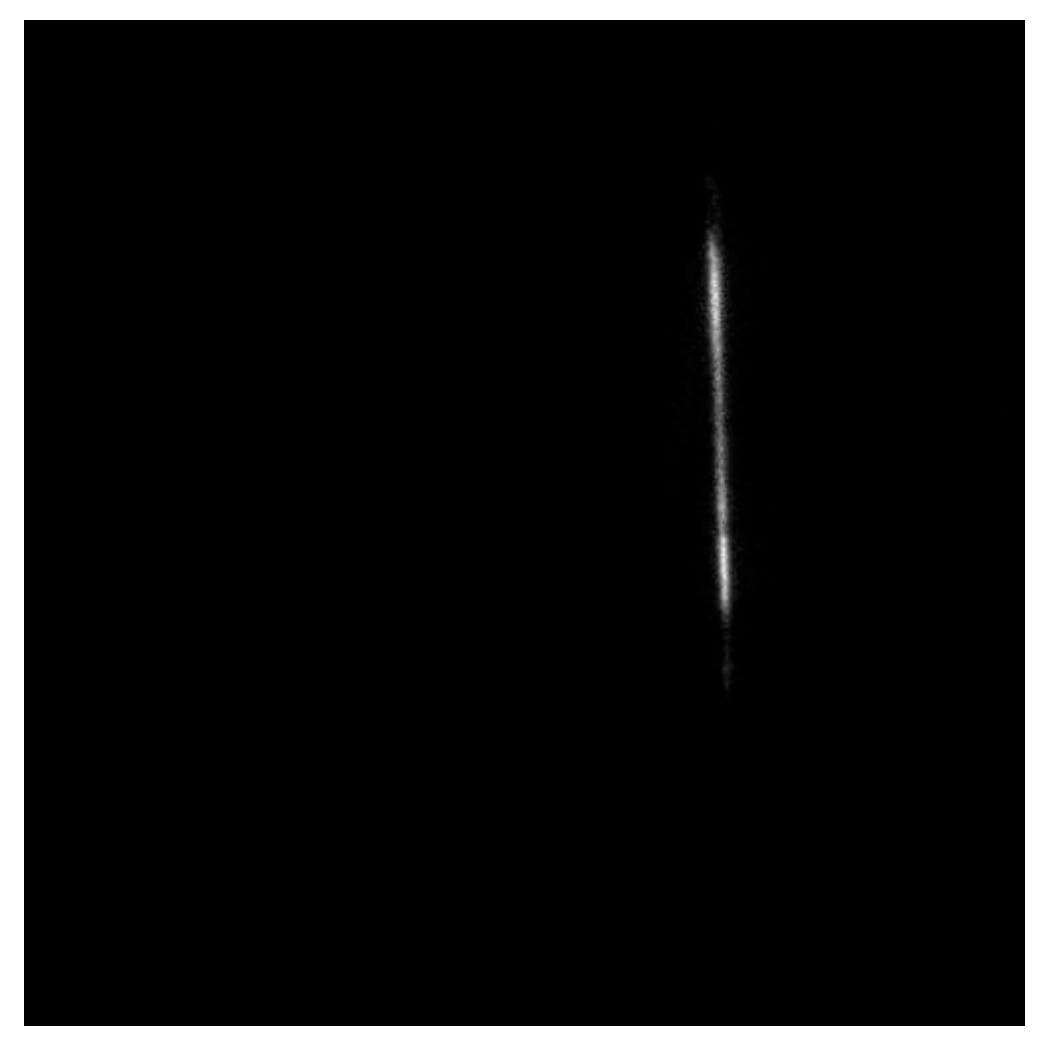

FIG. 6: With electron suppression on and all clearing electrodes off. (File 409220279

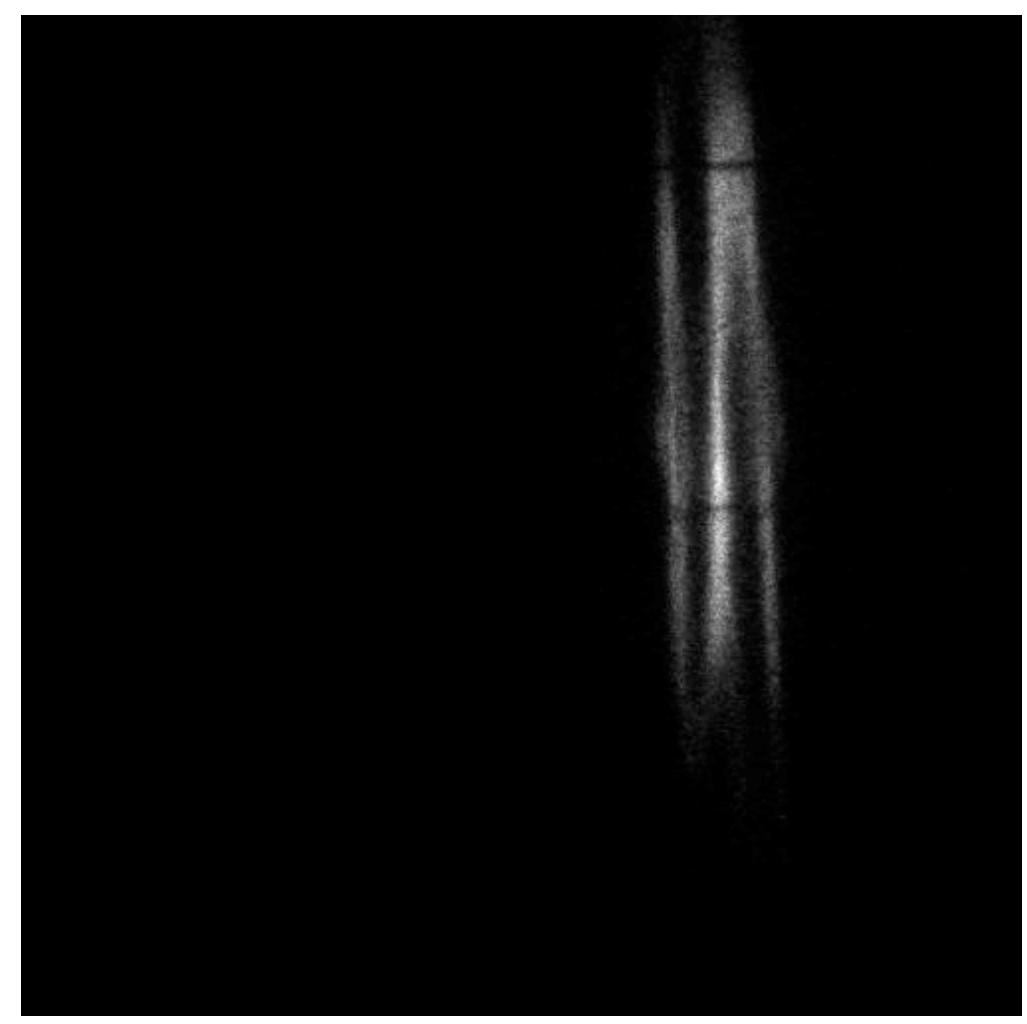

FIG. 7: With electron suppression off and all clearing electrodes off. (File 409220120). 


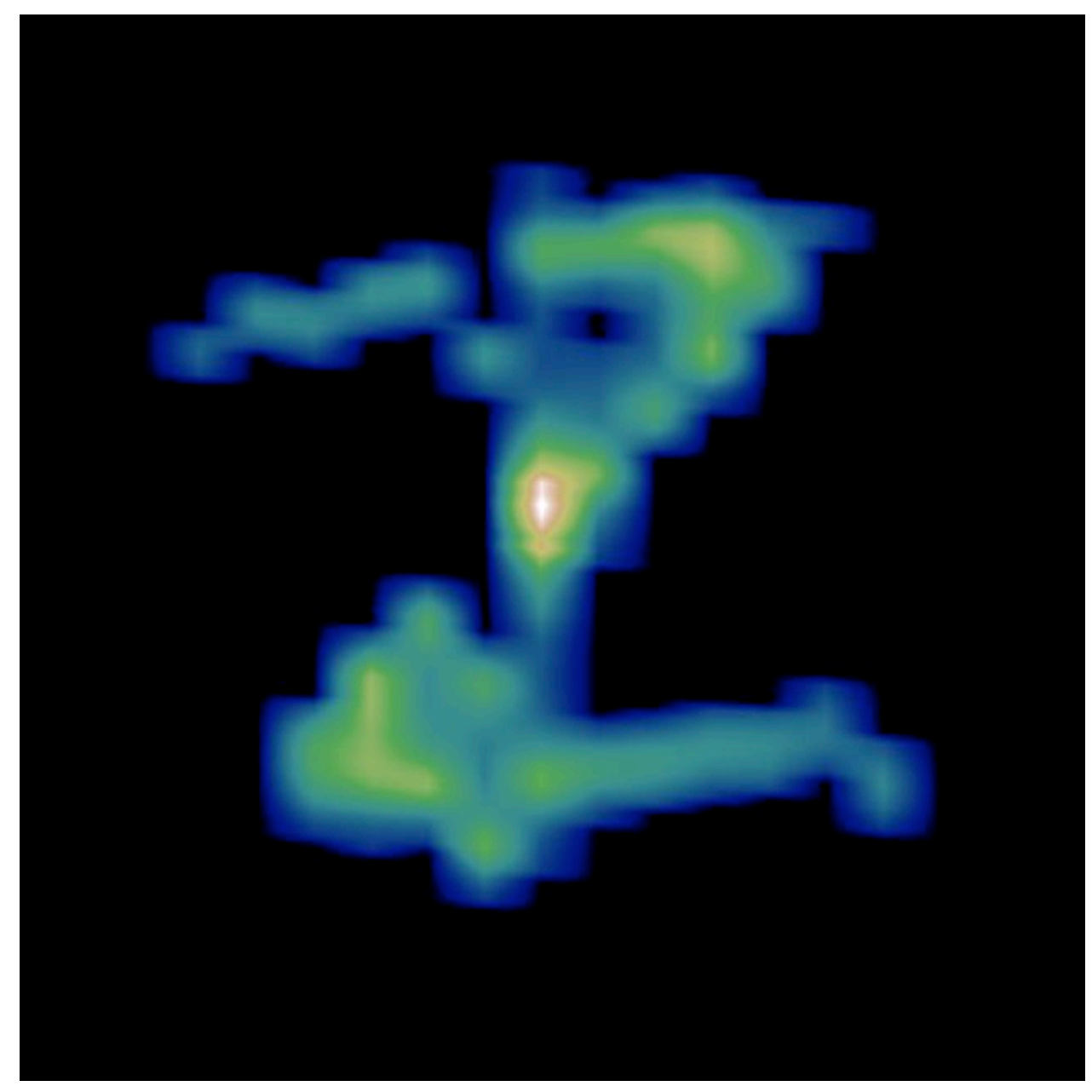

FIG. 8: Reconstruction of $x-x^{\prime}$ phase space from scan of slit data, with suppressor off and only the first clearing electrode biased, at $+9 \mathrm{kV}$. (File 409220222-257xx)

We measured the current to the clearing electrodes: those not biased drew little current, but clearing electrode (a) drew an electron current of about $8 \mathrm{~mA}$, Fig. 9. It is instructive to compare this current to the clearing electrodes with the beam current. The beam current is given in terms of the ion density $n_{b}$ and velocity $v_{b}$ and the beam cross sectional area $A$ as

$$
I_{b}=q n_{b} v_{b} A
$$

Similarly, the electron current from the end can be expressed in terms of the electron density $n_{e}$ and drift velocity $v_{d}$ as

$$
I_{e}=q n_{e} v_{d} A / 2
$$

Electrons drift through quadrupole magnets via $E \times B$ and $\nabla B$ (including curvature) drifts. 


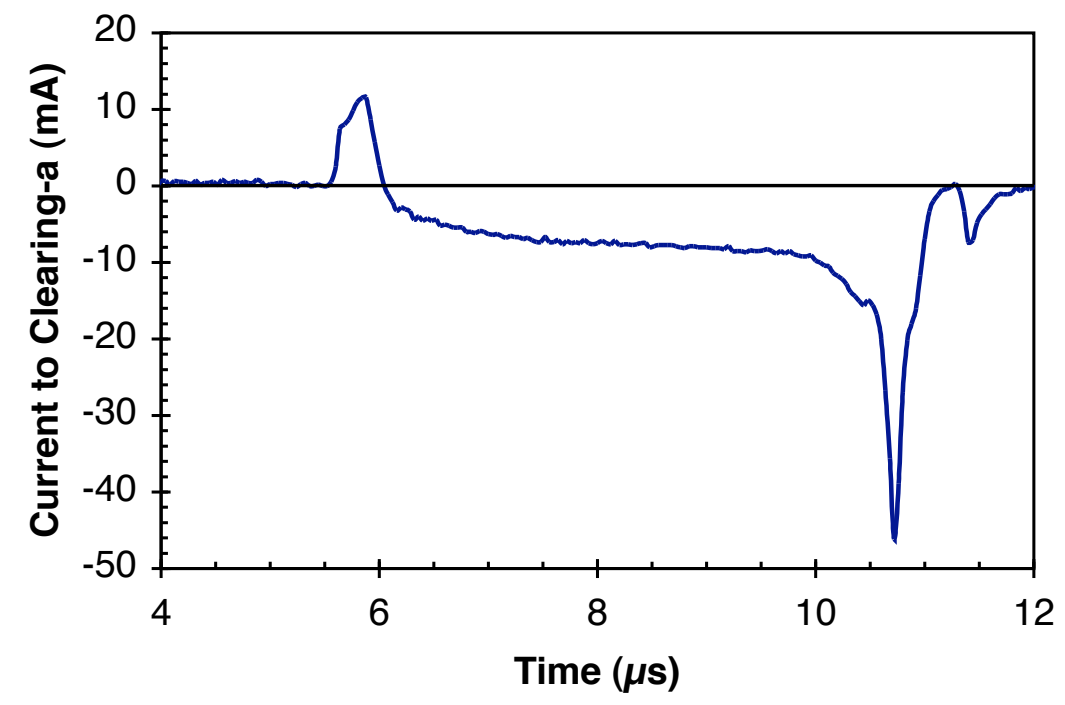

FIG. 9: Current to clearing electrode (a) biased to $+9 \mathrm{kV}$, the other clearing and suppressor electrodes at zero bias.

For electrons in a positive potential beam, these drifts are all in the same direction. The magnetic field direction of quadrupole magnets reverses in adjacent quadrants, resulting in the drifts also reversing direction. Therefore, electrons drift upstream (antiparallel to the beam) from the end in two opposite quadrants, and drift downstream in the other two quadrants, resulting in the area of electrons drifting upstream being $\mathrm{A} / 2$.

Dividing the second equation by the first, we obtain

$$
\frac{I_{e}}{I_{b}}=\frac{1}{2} \frac{n_{e}}{n_{b}} \frac{v_{d}}{v_{b}}
$$

The electron current in Fig. 9 is about $8 \mathrm{~mA}$ during most of the beam pulse, about 0.05 of the ion beam current which is $174 \mathrm{~mA}$. Simulations suggest that $v_{d} / v_{b} \sim 0.3$ (for the conditions simulated), which would correspond to $n_{e} / n_{b} \sim 0.3$.

With the suppressor biased, and all the clearing electrode biases turned off, the beam phase-space plot is much better as shown in Fig. 10. However, the kinks seen here are not present when the beam is measured upstream of the magnets. 


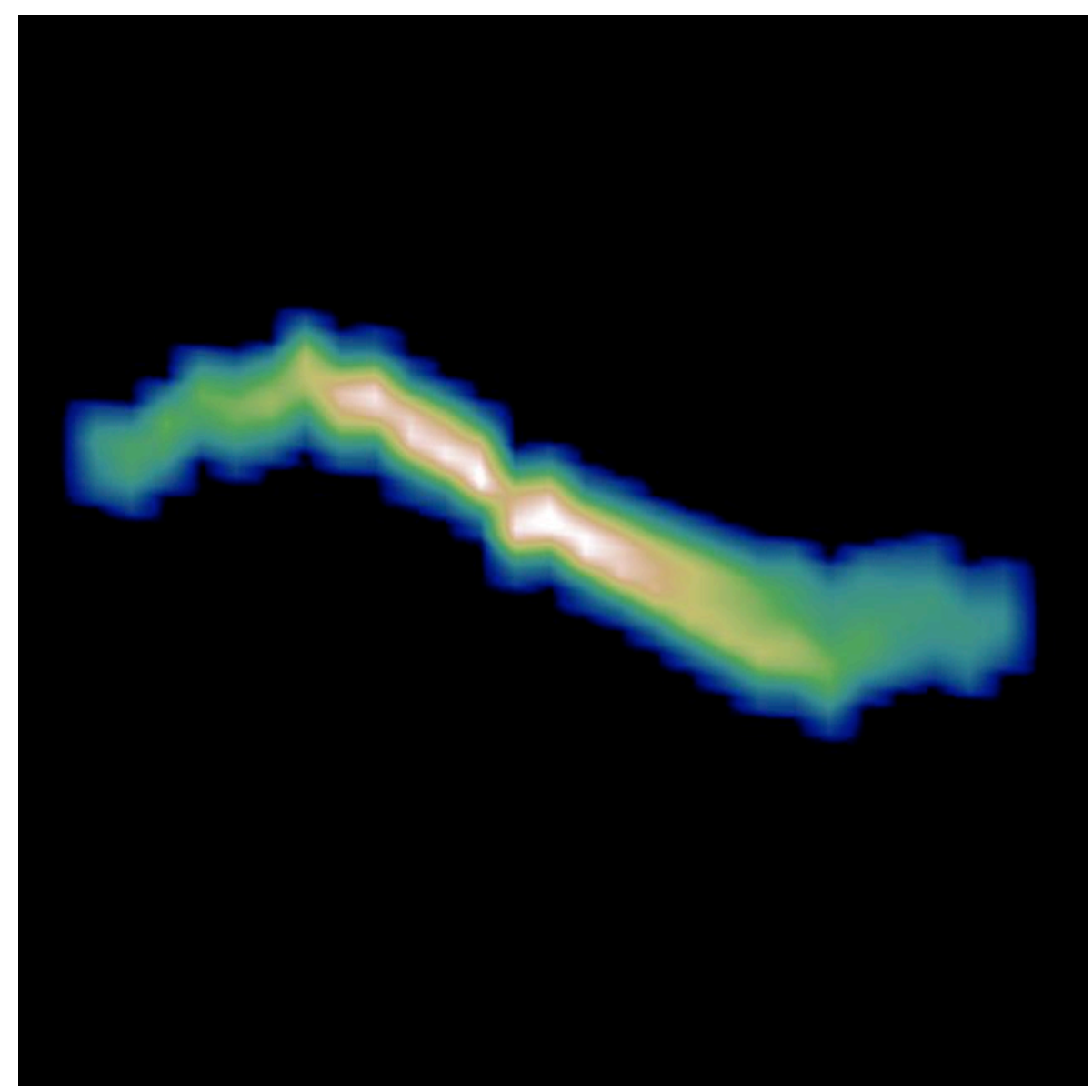

FIG. 10: Reconstruction of $x-x^{\prime}$ phase space from scan of slit data, with suppressor on and clearing electrodes off. (File 409220259-296xx)

\section{MODELING CAPABILITY IMPROVEMENTS}

Beginning with the 2003 Particle Accelerator Conference, we have been showing a chart of the ingredients we are assembling to enable self-consistent electron and ion simulation of accelerators in the WARP code. An updated version of this chart is presented in Fig. 11. The current status of each component is indicated by a color code. The changes over the 


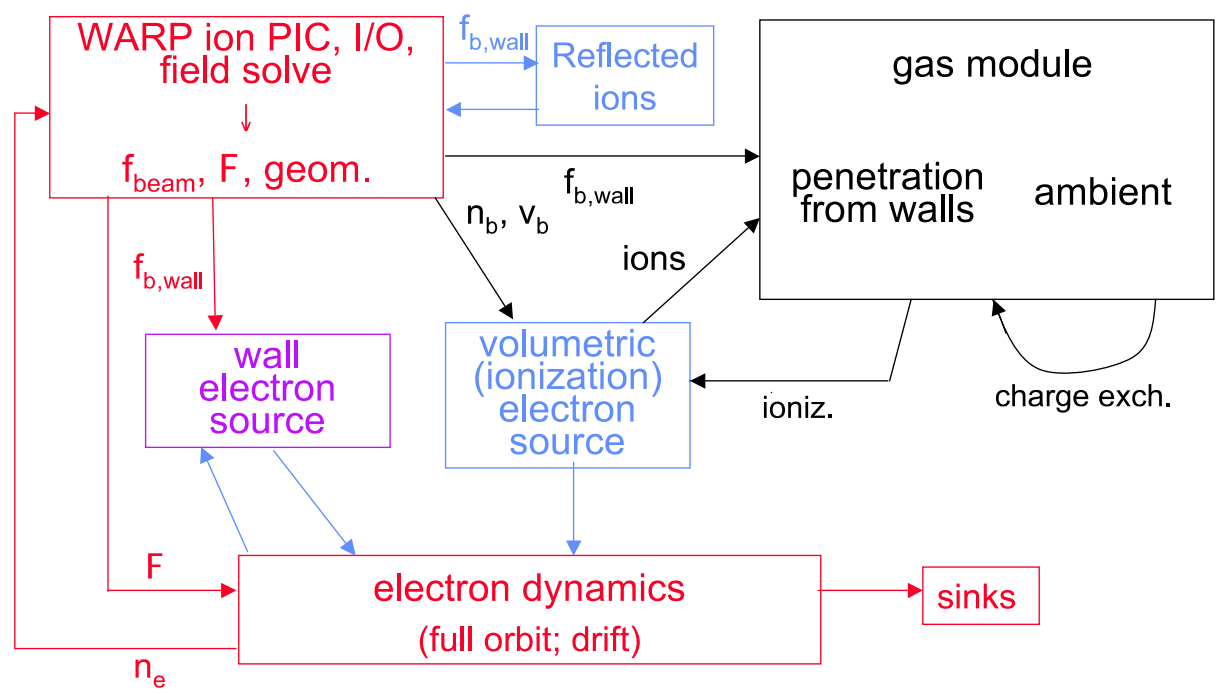

- Key: operational; implemented, testing; partially implemented; active offline development (as of 9/04)

FIG. 11: Chart of modules for self-consistent simulation of electrons and ions. Color coding indicates deployment status

past year are that the long-timestep electron dynamics capability ("interpolated drift" on the chart) is now operational; the volumetric electron source (from ionization of gas) is partially implemented; the wall electron source module is implemented and being tested; and models to describe neutral gas generation and evolution and a more accurate model of ion reflection at walls are in active development. These advances are each briefly described below.

\section{A. Interpolated mover}

Over the past year we have developed and done limited testing of a mover for electrons that interpolates between full electron dynamics and drift kinetics. The algorithm is briefly described in a paper submitted for publication [6], and will be more extensively detailed in an invited talk at the forthcoming American Physical Society Division of Plasma Physics meeting and the associated Physics of Plasmas paper. The algorithm builds upon the observation by Parker [7] that the conventional Boris particle advance scheme, when run with time steps large compared to the cyclotron period, continues to exhibit correct drift velocities, but causes particles to gyrate with a radius that is large compared to the physical gyro 

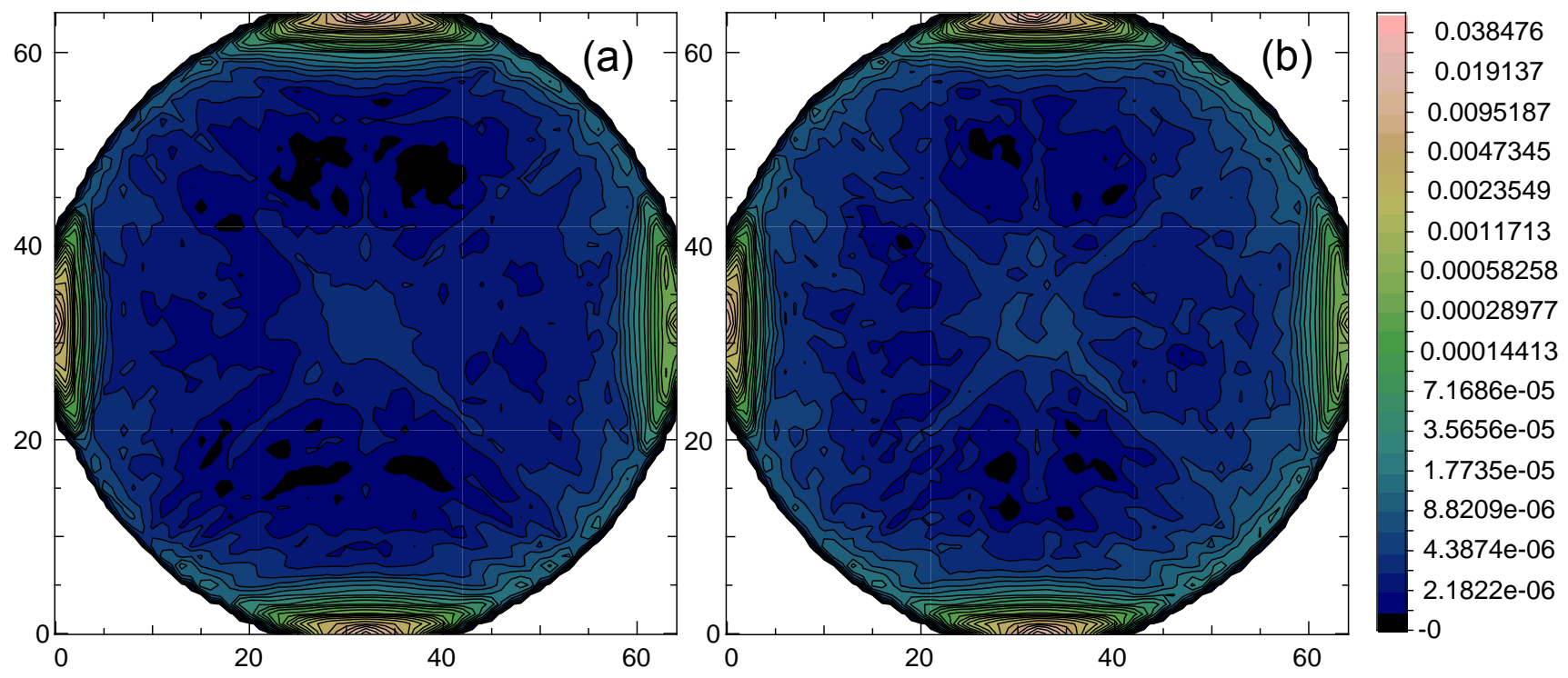

FIG. 12: Comparison of small-timestep (a) and interpolating-mover (b) simulations for electrons desorbed from walls. Timestep is 25 times bigger for interpolating mover.

orbit, and with a frequency that is lower than the physical gyrofrequency. Our interpolation scheme corrects the former deficiency, preserving a physical gyroradius, and is thus well suited for simulating particles that move through regions of strong, weak, and no magnetic field such as we have in HIF accelerators. The timestep is set by the requirement of resolving the next-shorter timescale of interest, which is the time for electrons to transit across the ion beam. The mover has been tested extensively with respect to single-particle dynamics; we find that it agrees well with small-timestep solutions for drift and bounce velocities and gyration radius, and also exhibits a transition from adiabatic (conserving magnetic-moment) to nonadiabatic (large jumps in magnetic moment) behavior at about the correct value of the particle energy (or equivalently, at about the correct distance of closest approach to the center of a quadrupole magnetic field). We have also tested the interpolated mover by comparing, with results from small-timestep simulations, its prediction for the spatial electron distribution produced by two different kinds of electron sources: (1) electrons desorbed from walls upon ion impact (as calculated from a WARP ion slice simulation, including a crude model of first-flight scattered ions); and (2) a source that is uniform out to a specified radius equal to the nominal ion beam radius (an approximate model for electrons produced by ionization of gas). The comparisons are shown in Figs. 12 and 13; the electron distributions in both cases are almost indistinguishable from the small-timestep results. 

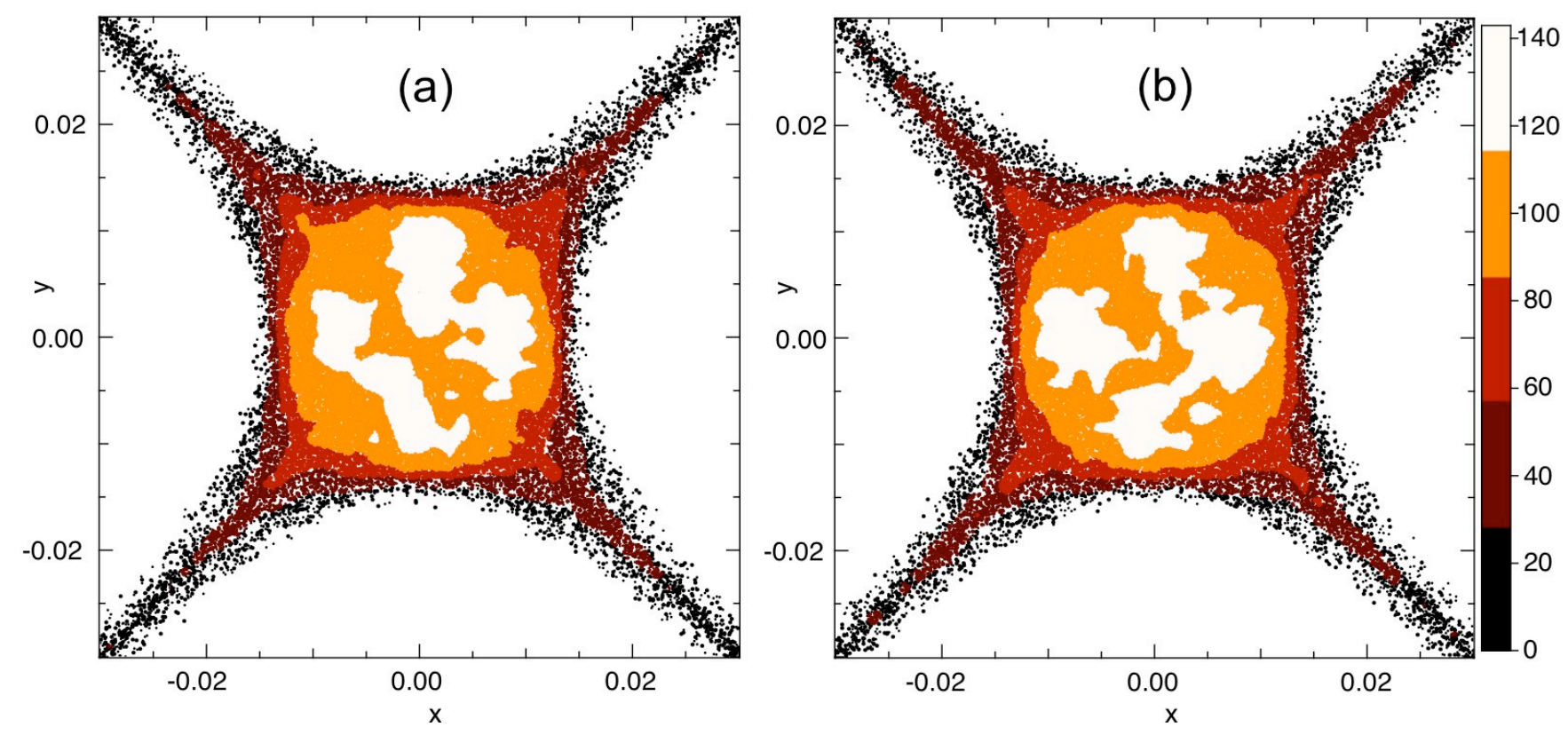

FIG. 13: Comparison of small-timestep (a) and interpolating-mover (b) simulations for electrons generated within a nominal beam volume. Timestep is 25 times bigger for interpolating mover.

Checks of the interpolating mover in a self-consistent simulation are just beginning; in fact the first real test is a limited version of the 3-D WARP simulation described in the next section. In that test, a single HCX magnet and the end tank were simulated using the interpolating mover (with a maximum timestep half the cyclotron period) and using the conventional mover (with a timestep 5 times smaller). Preliminary comparison of the results shows no evidence of numerical instability and nearly identical scatter and contour plots. Further tests are planned.

\section{B. Neutral gas module}

A neutral desorption model was developed at U.C. Berkeley in collaboration with the HIF-VNL. The scheme treats electronic sputtering induced by impact of energetic ions relevant to heavy ion fusion beams. The model includes angle-of-impact dependencies, and generates distributions of neutrals desorbed from the surface. The neutrals are returned as standard WARP particles, and can be followed in time using the standard WARP particle integration routines, using the standard WARP routines to compute surface interactions. WARP interpolation functions are used to weight the particles to an arbitrary mesh, and 
then to interpolate neutral density to continuum locations of the charged particles for use in a Monte Carlo collision scheme.

The model is partially complete. Presently the neutral desorption routines have been implemented in $\mathrm{C}$, and a Python interface for calling the functions has been created. Completion of the model will require integration of Python arrays for returning the created neutral velocities for use in WARP. The model can be easily extended to modify the created distributions and yield based on experiments currently underway in the HIF-VNL.

\section{Desorbed electron source module}

Tech-X Corporation, in collaboration with researchers from the HIF-VNL, is developing computer models of electron effects relevant to transport of high-current ion beams. These models include secondary electron production, ion-induced electron production, ion stopping in and scattering off beam pipe walls, and impact ionization of background gas.

The secondary electron production routines are those from the POSINST code from high energy physics. These routines accurately model both the number and energy distribution of secondary electrons produced when an ion collides with the beam pipe wall. Currently, the routines include parameters for stainless steel and copper surfaces. We are researching parameters for titanium nitride.

The ion stopping routines are based on the CRANGE routines developed for astrophysics. The rate of stopping is the basis for determining the rate of ion-induced electron desorption [4].

\section{Volumetric (ionization) electron source}

The impact ionization routines use cross sections based on fitting parameters from the Null Collision algorithm for efficient calculation of the number of collisions. Currently, the algorithms include parameters for electron impact ionization of hydrogen, helium, neon, and argon, and parameters for ion impact ionization of hydrogen, helium, neon, nitrogen, argon, carbon monoxide and carbon dioxide.

We used these routines to create a first estimate of the expected rate of electron production due to impact ionization in the end region of HCX (where the beam is incident normally 
on the end plate), and that estimate predicted the rate of electron production from impact ionization will be somewhat smaller than the rate of direct electron desorption (six electrons per incident ion).

\section{E. Reflected ions}

We are also developing models of ion scattering from metal surfaces. Because the beam velocity parallel to the beam pipe is much larger than the velocity perpendicular to the pipe, the ions strike the beam pipe wall at grazing angles. At these angles, the chance is high that the ions will scatter from the surface rather than penetrate into it. The SRIM code models such scattering events, but SRIM runs only on the Windows platform, and this precluded its direct use. To bridge the gap to other platforms, we have developed databases and access routines for using SRIM data in a cross-platform way. These databases and access routines are written in Python and therefore easily integrate with the WARP simulations.

\section{THREE-DIMENSIONAL, TWO SPECIES SIMULATION}

\section{A. Simulation setup}

The experimental setup described in Fig. 1 was reproduced in a WARP Python input file. Quadrupole fields are represented by a high-order multipole expansion while conductors (beam pipes and diagnostic plates) are embedded in the Poisson solver using the cut-cell method. The computational zone extends longitudinally from the exit plane of the last electrostatic magnet to the slit diagnostic plate "DHEX", located $26 \mathrm{~cm}$ downstream of the last magnetic quadrupole exit. Transversely, it extends from the beamline axis to four inches

both in $\mathrm{X}$ and in $\mathrm{Y}$. Fourfold symmetry was assumed (and a single quadrant simulated) to reduce the computation time. The transverse size was chosen to be approximately twice the larger aperture of the beam pipe in order to allow for possible large transverse excursions of electrons in the region between the last quadrupole and the diagnostic plate. The beam was launched at the exit of the last electrostatic quadrupole using the time-histories of the beam current, energy, transverse edge envelope dimensions and velocities, and emittances, all derived from experimental data. The detailed structure of the beam was not reproduced from experimental data; instead, a semi-Gaussian profile (flat in coordinate space, Gaussian in 
velocity space) was assumed. Beam ion macroparticles reaching the diagnostic plate DHEX generated 6 macroelectrons each, according to past measurements [5]. Since no secondary emission model has yet been implemented in WARP, a simple operational procedure was chosen: new macroelectrons were place a few cells (in this case 3) in front of the diagnostic plate with an initial random velocity corresponding to a temperature of $30 \mathrm{eV}$ and at the same transverse position as the incident ion.

\section{B. Simulation results and discussion}

In the absence of electrons, the 3-D simulation shows only a mild phase-space distortion; filaments appear at top and bottom, but are mere wisps; see Fig. 14. When selfconsistent electrons are allowed to enter the simulation, they are seen, at the end of the fourth quadrupole, to preferentially occupy two quadrants; see Fig. 15. In the figure, the elliptical pipe boundary is represented by the hash-marks separating the interior of the pipe from the exterior. The lengths of the hash-marks denote the subgrid-scale "cut-cell" boundaries used in WARP. A side-on view of the simulation (with greatly expanded transverse scale) is shown in Fig. 16.

The simulation as presented has several points of contact with the experiment: the electron density in the fourth quadrupole and in the end region is comparable to the ion density, and produces a significant distortion of the ion beam phase space, with a tendency toward a "Z"-shaped $x-x^{\prime}$ phase-space plot such as inferred from the experimental slit data (see Fig. 8). The simulation also indicates that, despite an electron density comparable to the beam density in the last magnet and the end region, the beam is able to propagate without significant loss to the end wall, again in agreement with the experiment. Finally, the electron density in the first three quadrupoles is approximately constant, as is also the case for the experiment.

However, there are several points of discrepancy: most notably, the observation from the experiment is that, when the suppressor ring is unbiased, the current collected at the biased clearing electrode is about the same regardless of which electrode is biased, and is consistent with an electron density comparable to the ion density traveling at a drift velocity (electric plus magnetic) of order of that which would be obtained from the beam potential. This suggests a substantial $\left(n_{e} \sim n_{b}\right)$ electron population in all quadrants downstream of 


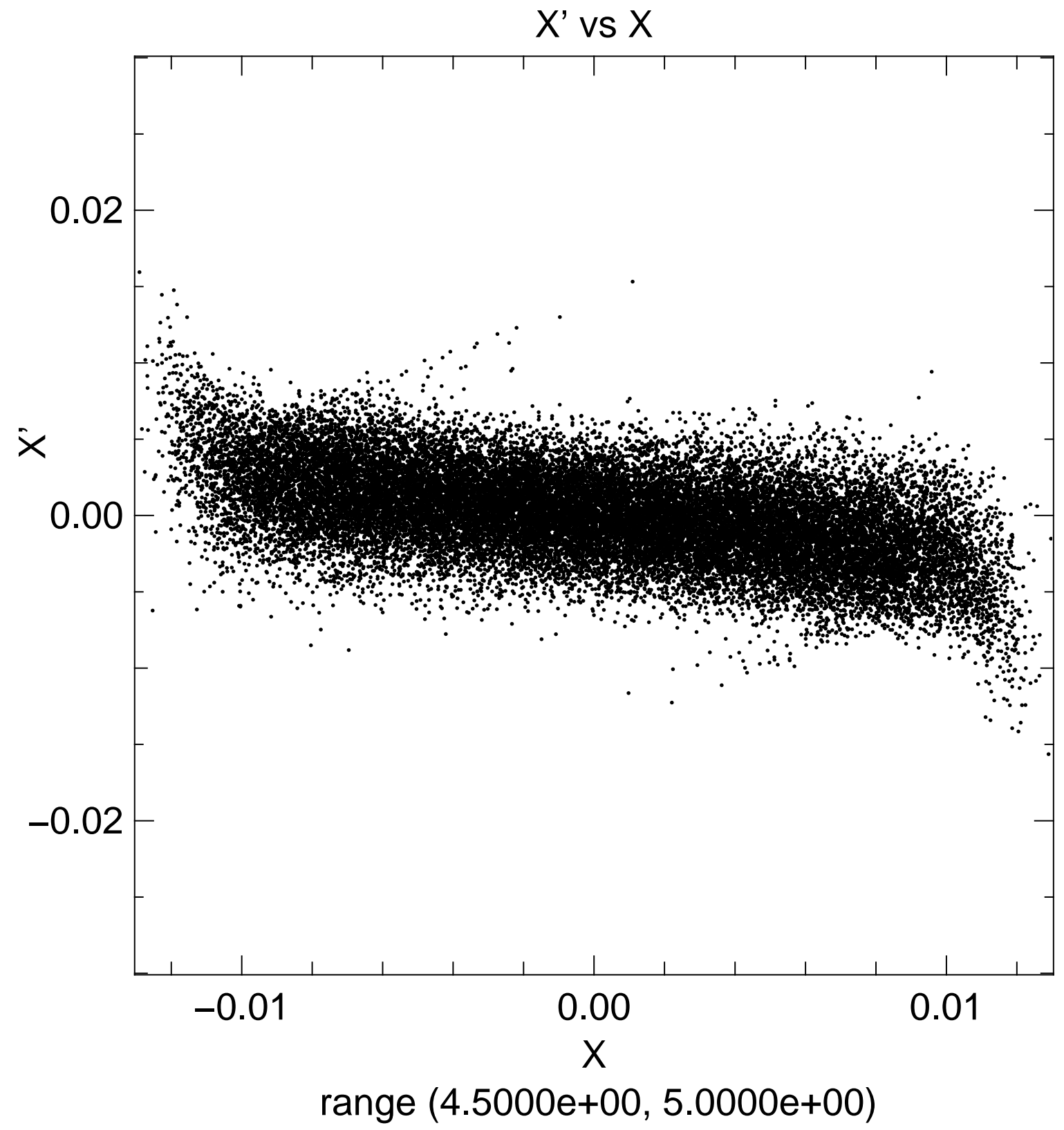

FIG. 14: $x-x^{\prime}$ scatter plot of particles at end of machine for 3-D simulation with no electrons, at $t=2 \mu \mathrm{s}$.

the biased clearing electrode. In contrast, the simulation shows a precipitous drop off in electron density as one exits the fourth magnet in the up-stream direction.

Examination of the $y-z$ particle scatter plot superimposed on equipotentials (Fig. 16), and examination of an $x-y$ scatter plot, color-coded to reveal the $z$ location of the particles (Fig. 17), suggests a likely explanation: the electrons drift outward until their bounce motion along field lines reaches the walls and they are lost. Because there is a grounded beam pipe 


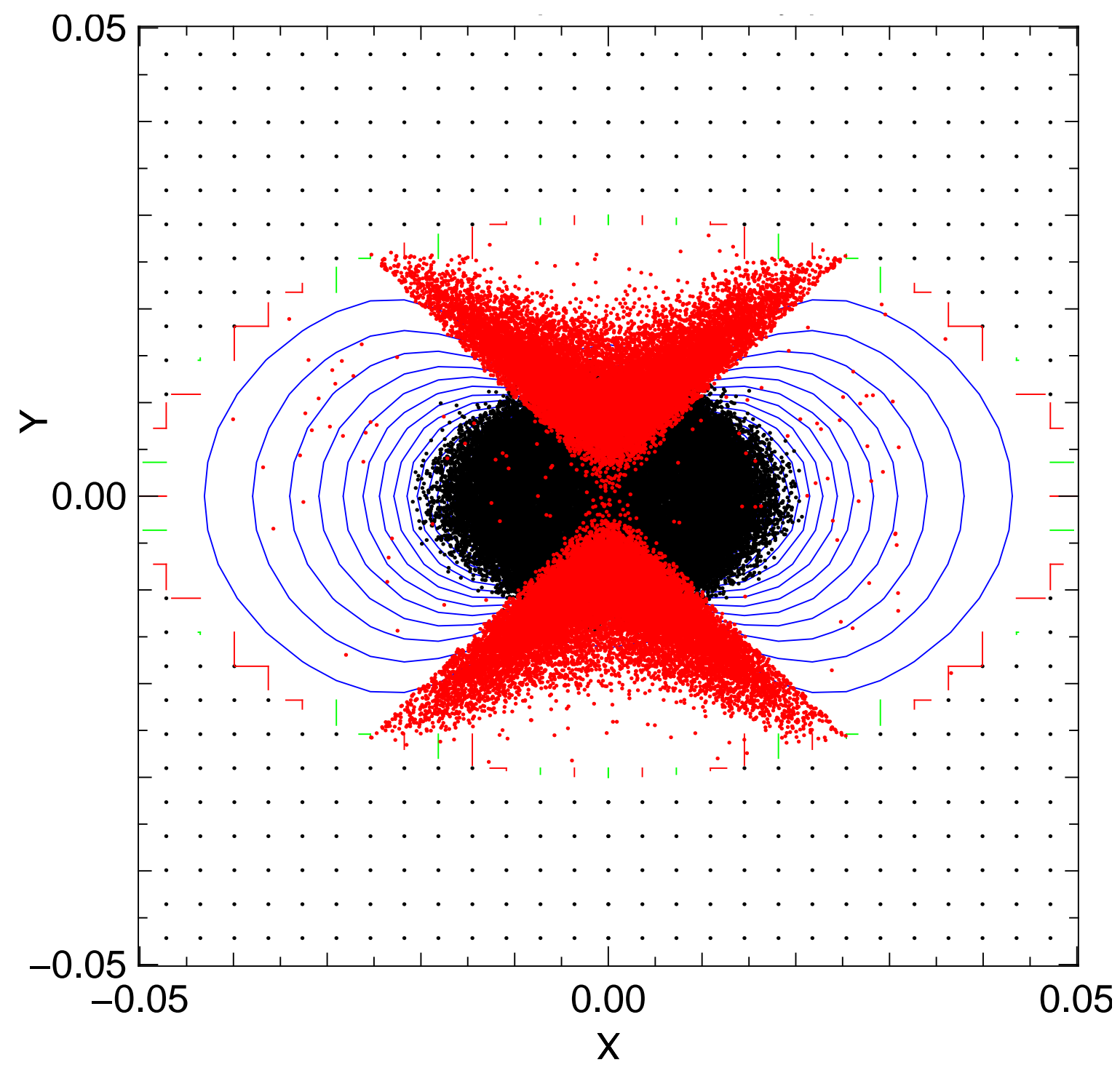

FIG. 15: $x-y$ scatter plot of particles at end of fourth quadrupole for 3-D simulation with electrons, at $t=4 \mu \mathrm{s}$. Electrons shown in red.

in the magnets but not in the gaps, the potential at a given radius is more negative in the gaps than in the quadrupoles. Consequently there is a $z$ (beam-wise) component of the electric field which produces a radial electric drift. The radial electric drift is proportional to $E_{z} B_{\theta} / B^{2}$. $E_{z}$ becomes appreciable in the fringe magnetic-field region at the end of the quadrupole, where $B$ is decreasing. Hence the $\mathbf{E} \times \mathbf{B}$ drift gets larger as an electron moves further into the fringe field. As enough electrons move outward in this region, they alter the charge distribution in a way that further enhances $E_{z}$ and the radial drift. It can be seen in the figure (by examining the left boundary of the "red" region) that the electron density 


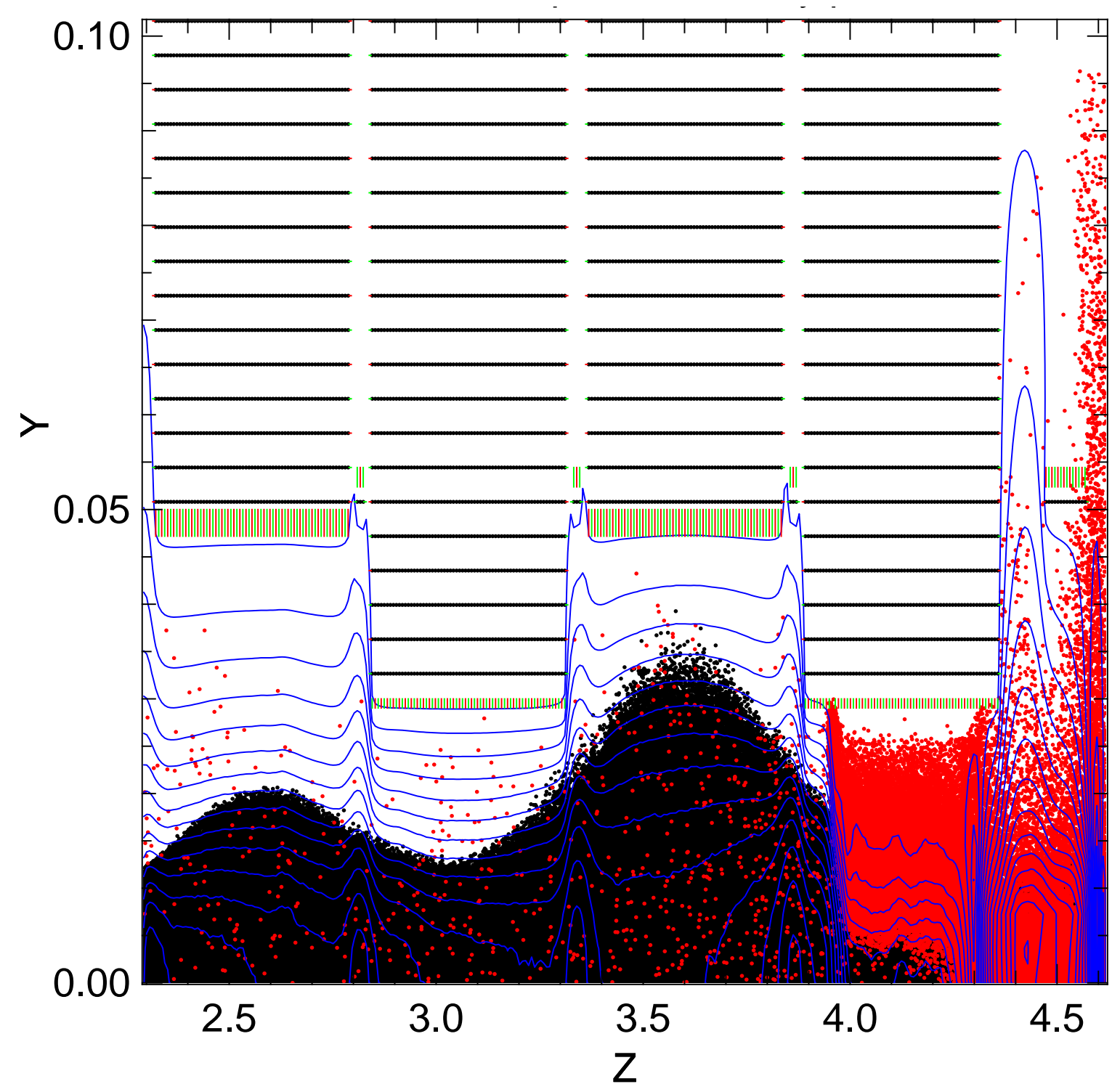

FIG. 16: Color-coded $y-z$ scatter plot of particles in 3-D simulation with overlay of equipotentials and conducting surfaces

tracks well with the equipotential contours, as one expects from the observation that the $\mathbf{E} \times \mathbf{B}$ drift moves particles on equipotential surfaces. Also, as one moves further into the fringe fields, the weakening of the magnetic field renders electrons more nonadiabatic: they begin to undergo pitch angle "scattering" from interaction with the magnetic field (see, e.g., Ref. [8]), with the result that, after some number of bounces, any electron which has enough kinetic energy to reach the wall will do so.

That the above mechanism should lead to electron loss is an artifact of the simple (absorbing) model for electrons used in the simulation. In reality there is an appreciable probability 


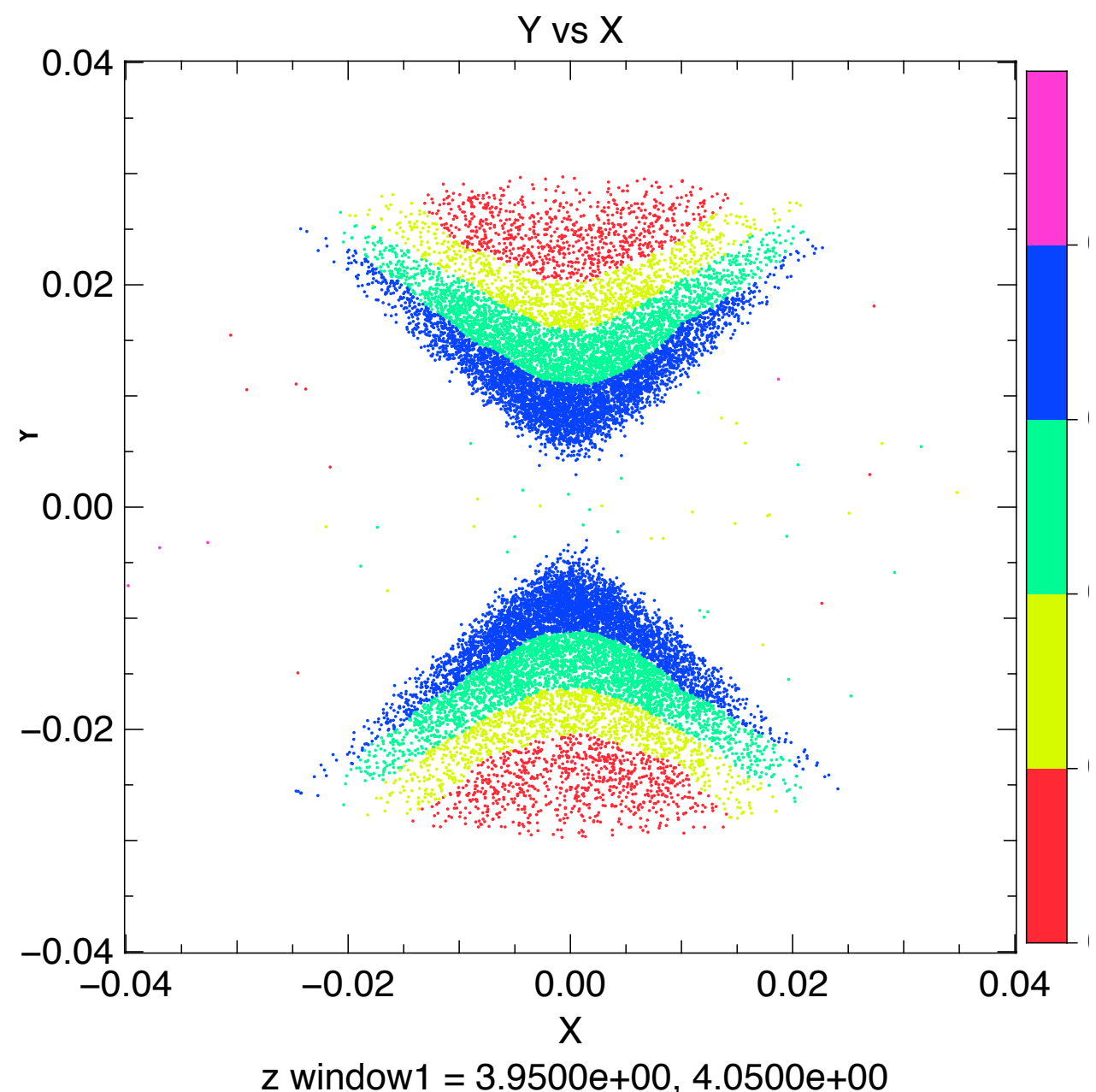

FIG. 17: $x-y$ scatter plot in 4th quadrupole's fringe field, color-coded to show z locations.

of re-emission of an electron, either through generation of secondary electrons or scattering or reflection. These processes are included in the POSINST models now in WARP, but have not been used to date. We are in the process of preparing runs that include this physics. In the mean time, we have done ion slice simulations with a specified electron cloud shape that anticipates the results we expect from the improved electron-wall interaction model; these are presented in the following section.

Another deficiency of the current simulation is the treatment of the ion-induced electron desorption at the end wall. Electrons in the simulation are born a few cells away from the wall and quickly form a virtual cathode, with the result that subsequent electron creation is at an artificially negative potential (relative to ground). As a result, electrons everywhere away from the virtual cathode region have an anomalously large kinetic energy. This can lead to quantitative errors in the electron density distribution anywhere; it can lead to 
qualitative errors in the calculation of electron loss to bounding surfaces around magnets. To fix this difficulty requires emitting electrons at the wall and resolving the resultant sheath potential, or deploying a reduced model of the near-surface region. This model must account for higher-than-one-dimensional effects, as the ability of electrons to leak out the sides of a virtual cathode region has an important impact on the character of the region. The need to develop such a model motivates the end-region simulations also described in the following section.

Another point of discrepancy between the simulation and the experiment is that the deformation of the electron phase space is less severe in the simulation than is inferred from the experiment, even for the experimental case where the clearing electrode between the third and fourth quadrupoles is biased so that there should only be electrons in the fourth quadrupoles. This aspect of the simulation is also inconsistent with the corresponding fixedelectron simulation described in the next section. We do not yet understand this discrepancy. A possible resolution with the experiment is that the simulation is done with an idealized (semi-Gaussian) beam injected just before the first magnetic quadrupole, whereas the phase space of the experimental beam is likely already somewhat distorted by mismatch of the electric and magnetic sections and other non-ideal effects.

\section{REDUCED CALCULATIONS}

We consider two classes of reduced simulations which address current deficiencies in the full 3-dimensional simulation. The first, ion slice simulations with prescribed electron clouds, are aimed at predicting the effects on the ion beam for the electron distribution we anticipate from the corrected 3-D simulation. The second, fine-scale simulations of the end region, are aimed at developing insights into and a model for the emission from the end-wall, including the effects of any virtual cathode that forms.

\section{A. Fixed-electron slice simulations}

We report here on a series of simulations with a fixed distribution of negative charge in and between magnets, chosen to be representative of the electron cloud distributions we expect in the experiment and ultimately in the self-consistent simulations. 
We begin with a discussion of the electron distribution to be expected in this system. Around six electrons are emitted from the end wall for every incident ion, and these electrons will fall through the beam potential and travel upstream (relative to the ions) with a speed of order $\left(2 e \phi_{\text {beam }} / m_{e}\right)^{1 / 2}$. (The suppressor ring near the end-wall is negatively biased or grounded to repel electrons emitted from the slit plate) But they are then largely reflected by the quadrupoles (in two quadrants of the quadrupoles, the electric drift will not admit electrons; in the other two, electrons are admitted slowly relative to their speed in the end region). Consequently we expect the electron density $n_{e}$ in the end region to build up to a level comparable to the beam density $n_{b}$, specifically until the beam potential is reduced to the point that electrons are no longer well-confined by the beam potential and are lost at the same rate as they are injected. A similar argument says that electrons should populate the two allowed quadrants of the quadrupoles (the quadrants where the drifts admit electrons) up to a density again of order of the beam density. For the case where the clearing electrode between the third and fourth magnets is biased positive, the electron density will then be of order of the ion density in the two quadrants of magnet 4 where the drift is upstream, and approximately zero in the other two quadrants and everywhere upstream of magnet 4 . For the case where the clearing electrode between magnets 3 and 4 is not positively biased, we expect that the gap in this region will, in time, fill up to $n_{e} \sim n_{b}$ for the same reason as in the end tank, and that the remaining quadrants of magnet 4 will then also fill up.

Hence our general picture is that if a particular clearing electrode is turned on, the quadrant immediately downstream of it will have two quadrants appreciably populated with electrons, and two empty quadrants, whereas magnets further downstream will be populated (with $n_{e} \sim n_{b}$ ) in all four quadrants, and all gaps downstream from the biased clearing electrode will be filled with $n_{e} \sim n_{b}$. All magnets and gaps upstream from the biased clearing electrode can be taken as empty. The 3-D simulations suggest that electrons are concentrated along hyperbolic magnetic field lines that are at most around $2 \mathrm{~cm}$ from the quadrupole cusps. For the case simulated with the 3-D code, which has only upstreamdrifting electrons in the 4th quadrupole, there is a depletion of electrons in the immediate vicinity of the quadrupole cusp (the straight field lines along the diagonals), which we tentatively attribute to nonadiabatic magnetic-moment scattering and its accompanying cross-field diffusion into quadrants which expel electrons back into the end tank. Hence, our model for the quadrupole adjacent to the biased clearing electrode is hyperbolic electron 

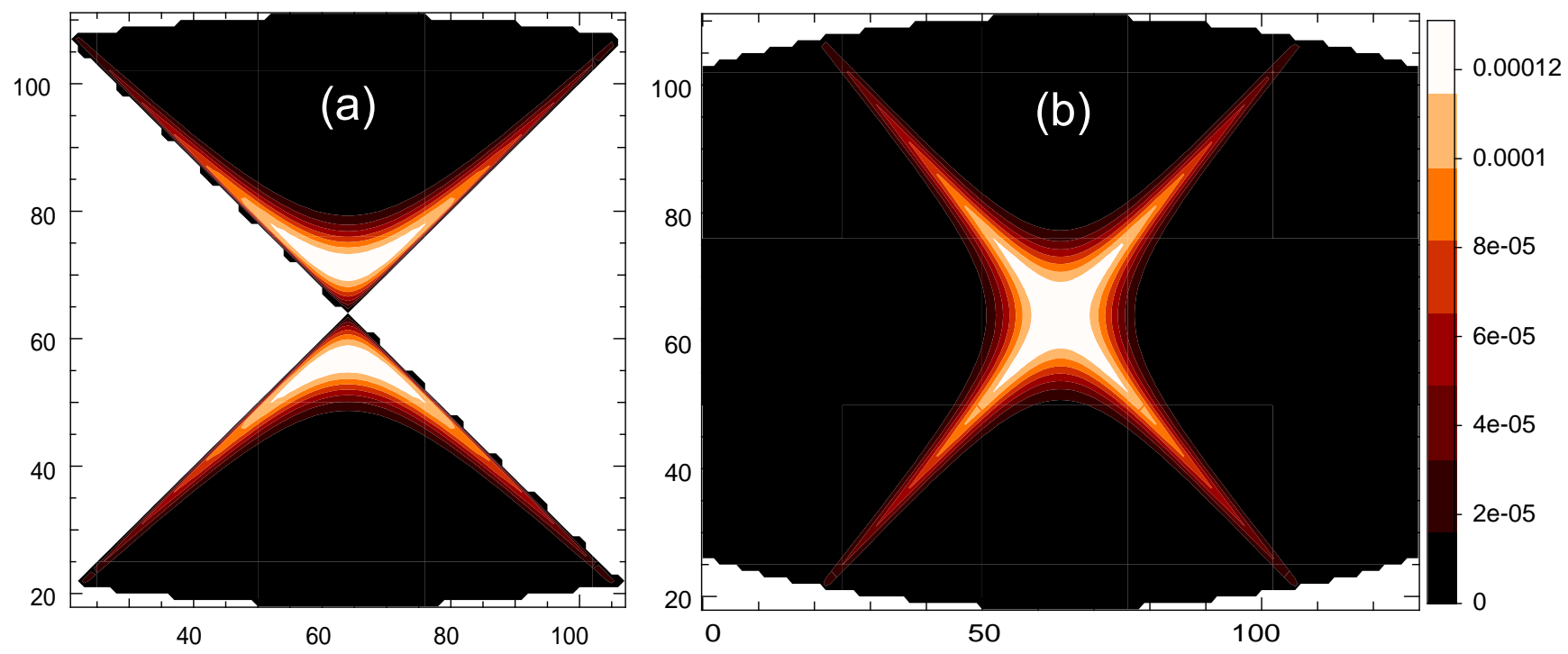

FIG. 18: Model electron distribution functions in transverse $(x, y)$ plane: (a) in magnet where only two quadrants are filled, and (b) in magnets where four quadrants are filled. Note that in the color palette used, white denotes both peak and zero density, while black denotes a small positive density.

slivers in two quadrants, with the density vanishing on the quadrupole cusp line. In other quadrupoles the electrons live on back-to-back hyperbolic slivers on opposite sides of the cusp line, with no gap in between. Specifically, in the latter case we take $n_{e}=N(x, y)$, with $N=n_{0} \exp \left(-r^{2} / r_{0}^{2}\right) \exp \left(-|\psi|^{1.5} / L_{h}^{3}\right)$ where $\psi=x^{2}-y^{2}, r^{2}=x^{2}+y^{2}$, and $r_{0}$ and $L_{h}$ and $n_{0}$ are constant parameters, and in the former case, we take $n_{e}=|\psi|^{1 / 2} N(x, y) \theta(\sigma \psi)$, where $\theta$ is the Heaviside function and $\sigma$ alternates between +1 and -1 in successive quadrants (such that $n_{e}$ is non-zero in quadrants where the electric and magnetic drifts for a circular beam are upstream). The two kinds of distributions are illustrated in Fig. 18. In between magnets, the electron distribution is a cylindrically symmetric Gaussian with a mean radius of $1 \mathrm{~cm}$.

We show, in Figs. 19, 20, 21, and 22, scatter plots for transverse position, velocity, and $x$ and $y$ phase space for three situations of experimental interest: (1) no electrons; (2) electrons in only the fourth quadrupole, and in only two quadrants, corresponding to the experiment with the third clearing electrode biased; (3) electrons in the second, third, and fourth quadrupoles but only in two quadrants of the second quadrupole, corresponding to the experiment with the first clearing electrode (between the first and second quadrupoles) biased; and (4) electrons in all quadrants of all four quadrupoles, corresponding to the 
experiment with none of the clearing electrodes being biased; in this case electrons should bounce off of the last electrostatic quadrupole (which is negatively biased) further upstream and return to fill the first magnetic quadrupole. In each case the peak electron density is equal to the ion density (and the average is somewhat less). What is evident from these simulations is that there is a noticeable effect on the ion phase space even for one quadrupole filled with electrons, but the size of the effect increases with the number of quadrupoles filled. For electrons in one quadrupole, $x^{\prime}$ versus $x$ indicates a small degree of multi-valued character, involving a relatively small number of ions, and a noticeable "z-ing" (bending) of the main part of the ion distribution, similar to what is observed in the three-dimensional simulation. With no electrons, the small amount of multivaluedness, involving the last few ions (black in the figure) persists, but the main part of the distribution is quite straight. We recall that multivalued $x^{\prime}$ is the condition that leads to multiple stripes in the slit experiments

described in Sec. II. Also we call attention to the similarity of the phase space plot for the simulation with four quadrants having electrons (Fig. 22) and the phase plot reconstructed from the experimental slit data shown earlier, Fig. 8.

These simulations reaffirm that the electron cloud levels that are inferred from the experimental data are consistent with sizeable changes in the ion phase space as indicated by the data. They also indicate that multiple images from slit experiments could be obtained, as observed on HCX.

We have not attempted a more quantitative comparison at this point because to do so would be premature. The 2-D model employs only an approximate description of the electron cloud shape; electron dynamics are not included; and the ion beam injection distribution is likely too idealized. We will undertake such comparisons when we have 3-D simulations in hand that address the deficiencies noted above.

\section{B. Detailed simulations of end region}

In this section, simulations of virtual cathode formation, performed in a collaboration between U.C. Berkeley and the Virtual National Laboratory, are described. The goal is to investigate the formation of a virtual cathode due to emission of secondary electrons generated by ion impact at the end wall. In the large WARP simulations, it is computationally expensive to resolve the spatial features of the virtual cathode, which have a scale length 

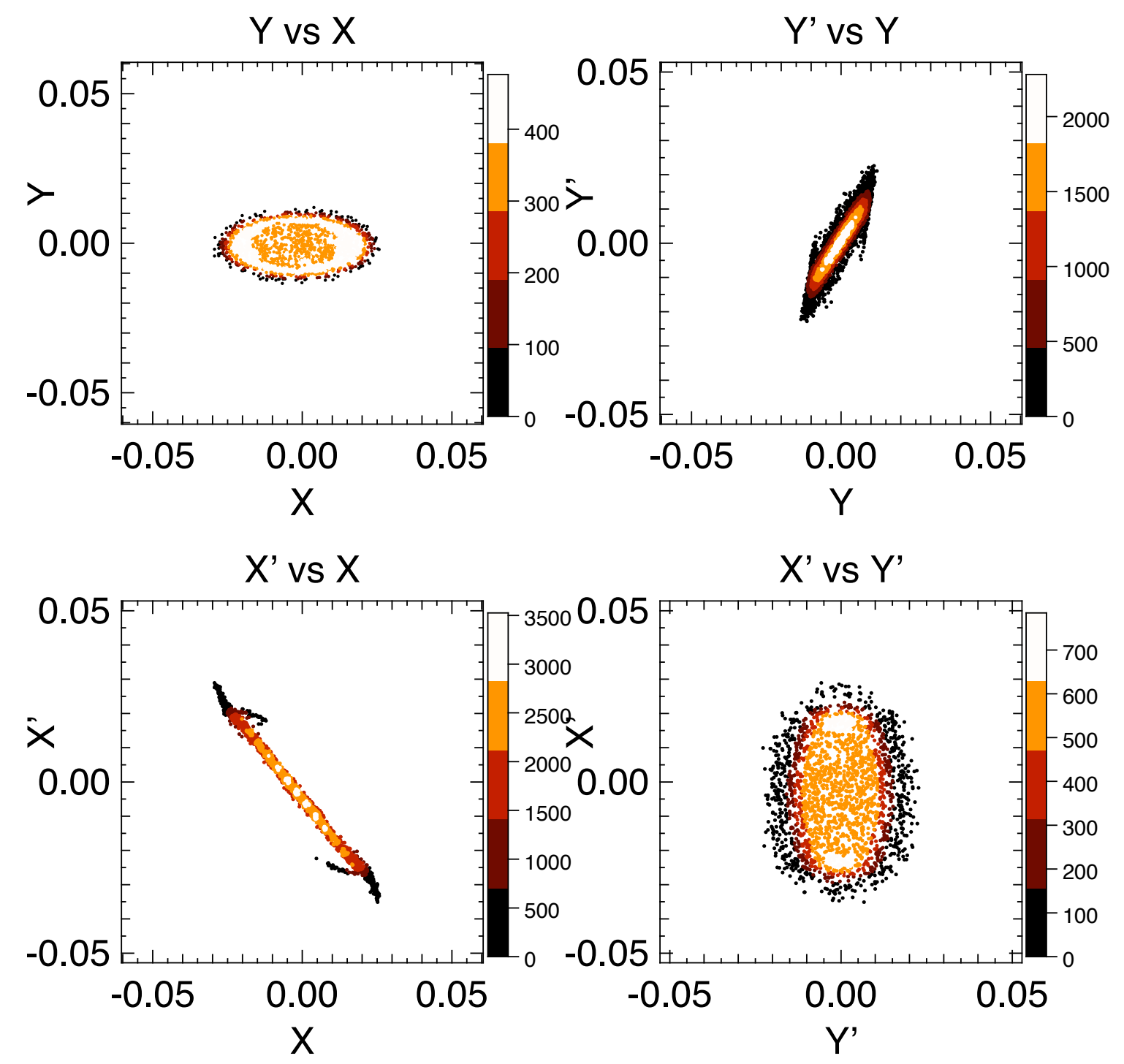

FIG. 19: Ion scatter plots near exit of last quadrupole for slice simulation with no electrons

of order the local electron Debye length. Using the axisymmetric XOOPIC code [9], we investigated the effects of resolving the virtual cathode.

A $972 \mathrm{keV}, 174 \mathrm{~mA}, 22 \mathrm{~mm}$ radius beam of $\mathrm{K}^{+}$ions propagating in a grounded right circular cylinder of length $26 \mathrm{~cm}$ (from the final quadrupole) and radius $11 \mathrm{~cm}$ impacts the end wall. Secondary electrons are generated with a secondary coefficient of $\gamma=6$, and a temperature of $T_{s e}=30 \mathrm{eV}$. The high resolution simulation used $\Delta z=1 / 3 \mathrm{~mm}$, and $\Delta r=0.55 \mathrm{~mm}$, and the low resolution simulation increased the cell sizes by a factor of 5 . A timestep of $\Delta t=0.2$ ns was used in both cases, sufficient to resolve the electron plasma frequency at the potential minimum. 

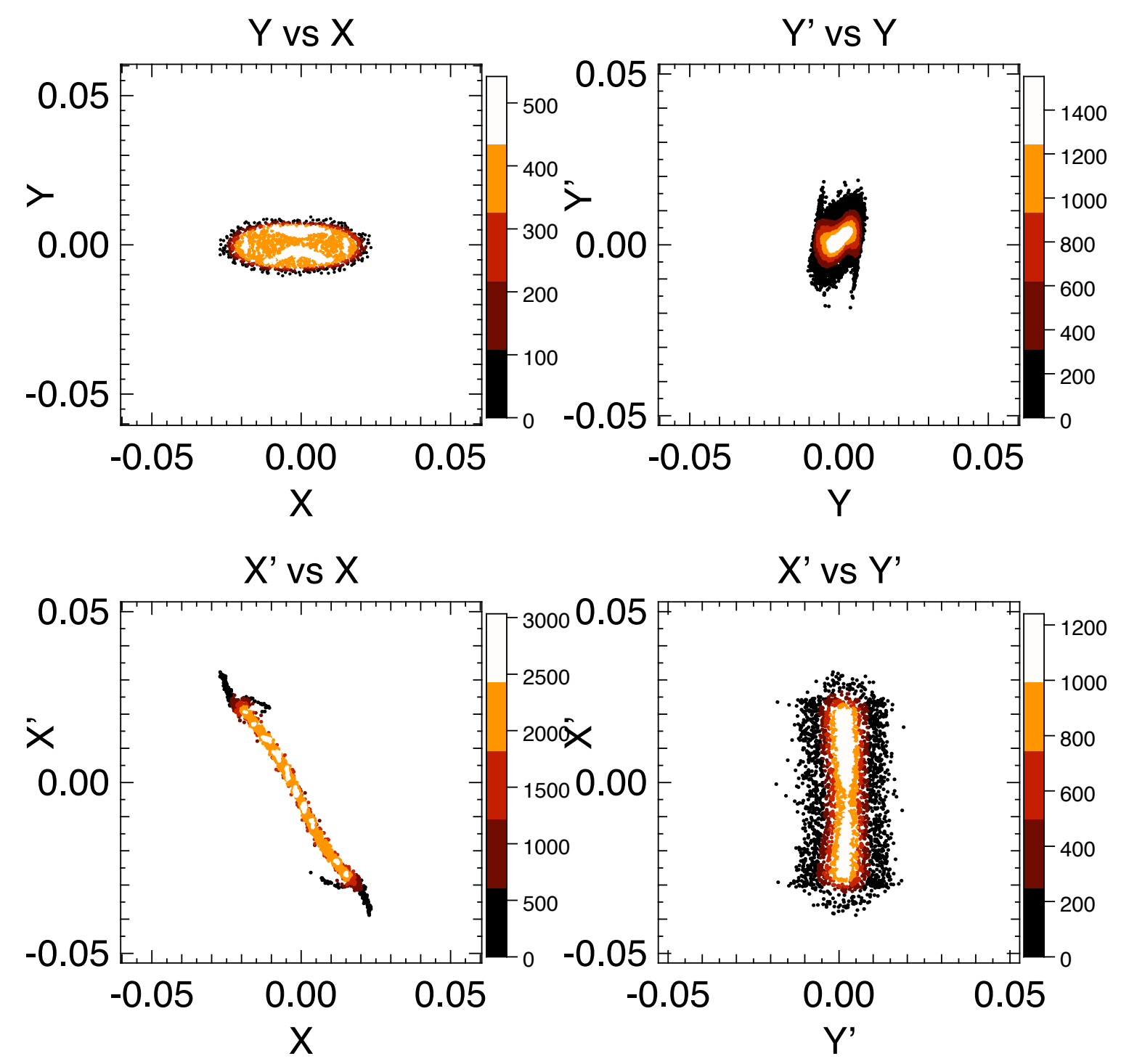

FIG. 20: Ion scatter plots near exit of last quadrupole for slice simulation with fixed electrons in two quadrants of fourth quadrupole

The temporal evolution of the number of particles is shown in Fig. 23. The ion density rises nearly linearly to its steady state value within an ion transit time, and then electrons are emitted from the impact surface. The rapid rise in the number of electrons is slowed by the local increase of the negative space charge as the virtual cathode forms. Those electrons born with sufficient energy to make it past the virtual cathode barrier accelerate into the positive beam potential, with some impacting the other side. Note that axial energy is not conserved since the unmagnetized electron beam is focused and defocused by the ion beam potential, and interacts weakly with the ion beam. Consequently, some electrons scrape off 

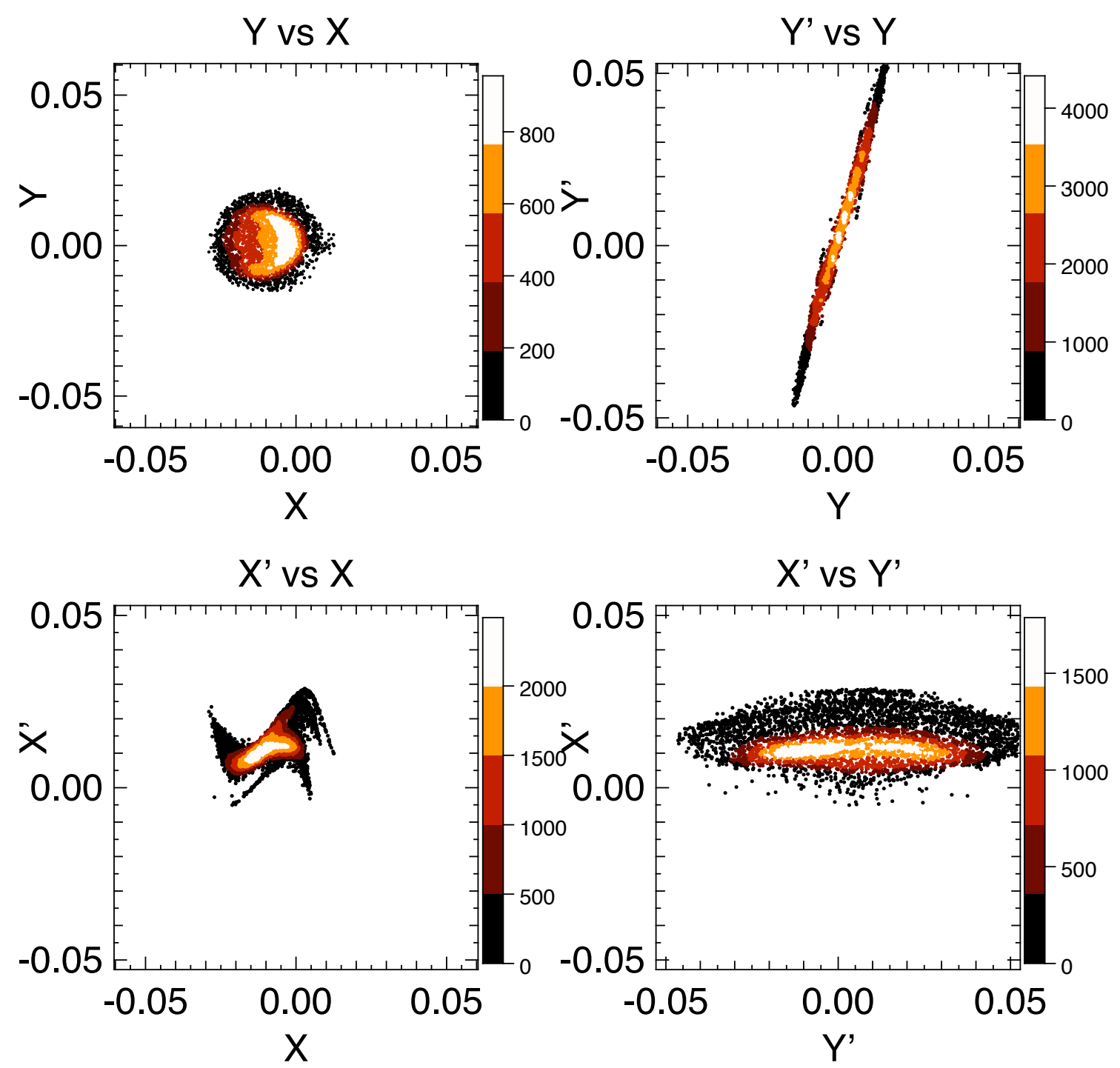

FIG. 21: Ion scatter plots near exit of last quadrupole for slice simulation with fixed electrons in two quadrants of second quadrupole and all quadrants of third and fourth quadrupoles

on the ion-emitting wall, and others are turned near the wall and circulate. Those that circulate appear to undergo a two stream instability with the counterstreaming electrons which leads to the buildup of electrons between 0.2 and 1 microsecond. During this buildup, the electron phase space hole collapses and fills in (Figs. 24 and 25), and the beam potential is gradually reduced (Figs. 26 and 27). This has the further effect of reducing the space charge current limit at the virtual cathode, causing the depth of the virtual cathode to increase and consequently reject more secondary electrons.

The high and low resolution simulations are qualitatively similar, but the low resolution 

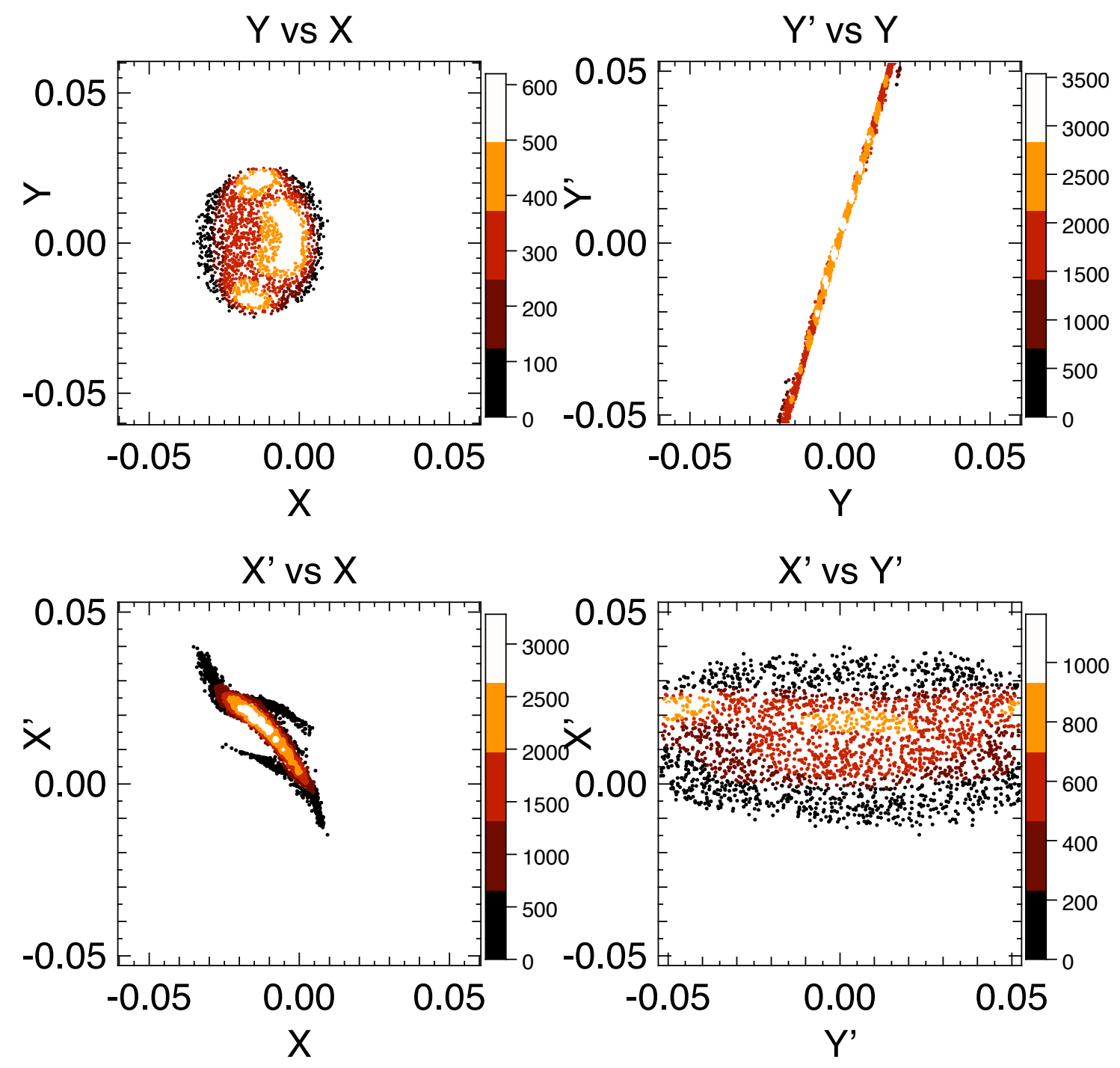

FIG. 22: Ion scatter plots near exit of last quadrupole for slice simulation with fixed electrons in all quadrants of all four quadrupoles

case results in about $2 \%$ more electrons than ions. While this seems minor, note that because the electrons and ions nearly neutralize each other, this amounts to nearly $100 \%$ difference in net space charge density in the volume. Comparing the virtual cathode potentials, Figs. 28 and 29 , the high resolution case results in a potential of about $-24 \mathrm{~V}$, while the low resolution case jumps rapidly in time between -25 to $-50 \mathrm{~V}$. The width of the virtual cathode in the high resolution case is about $1.67 \mathrm{~mm}$ ( 5 cells), while the low resolution case forces the width to a single cell, at $2 \mathrm{~mm}$. Note also the more rapid rise in the potential for the low resolution case. 


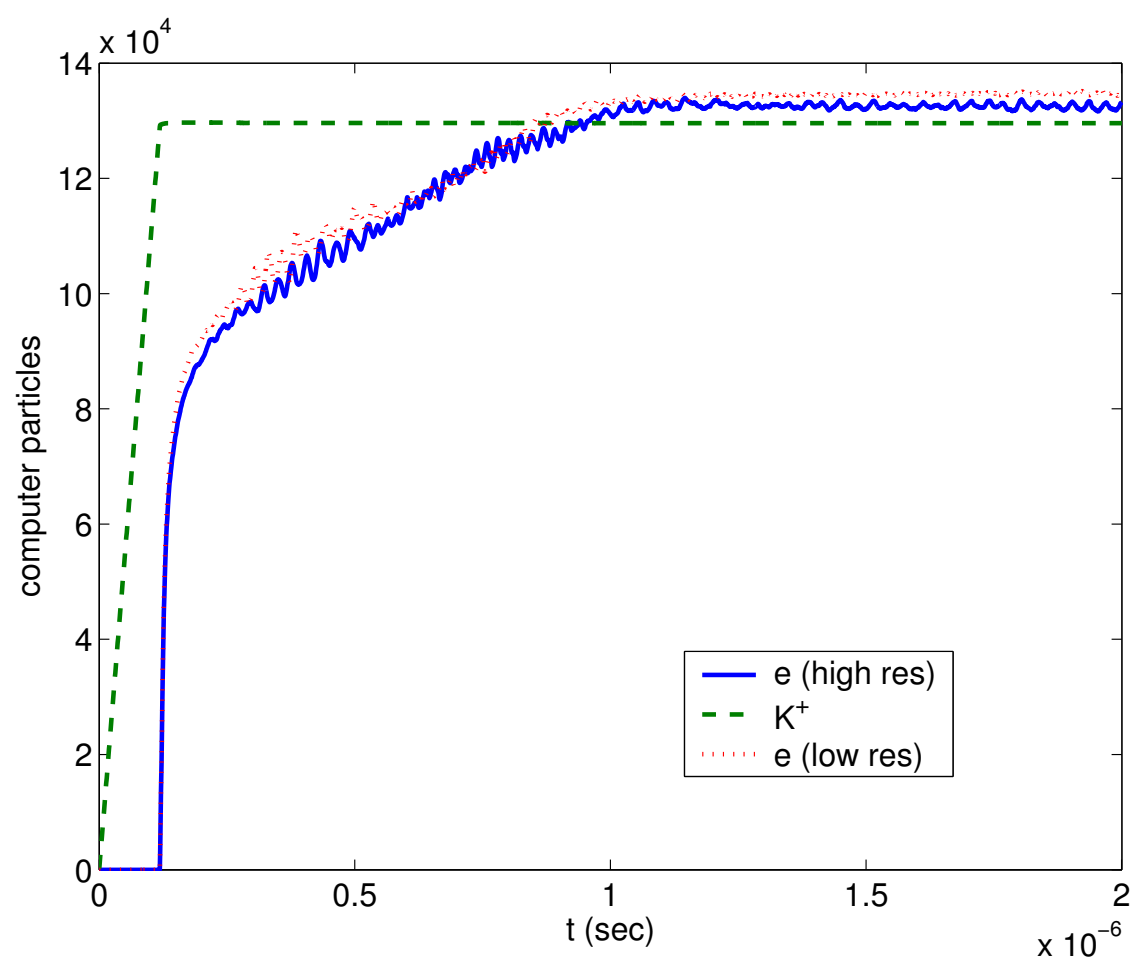

FIG. 23: Number of computer particles versus time.

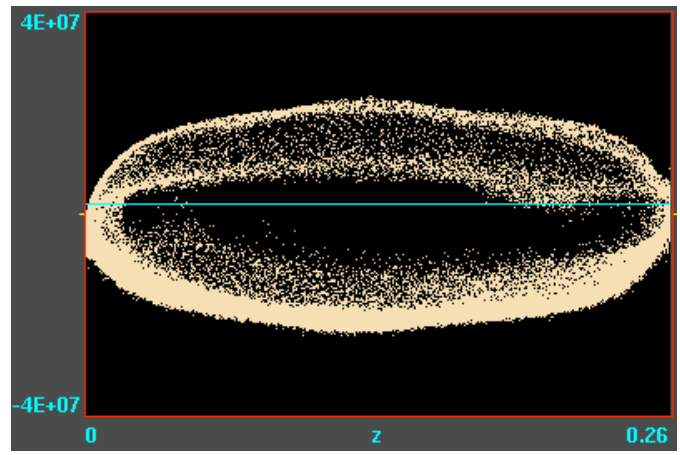

FIG. 24: Axial velocity phase space for the high resolution case at $0.2 \mu$ sec. Abscissa is $z(\mathrm{~m})$, ordinate is $v_{z}(\mathrm{~m} / \mathrm{s})$.

The study of the virtual cathode formation, and means of getting the macroscopic physics correct for the election population without the computational expense of resolving the virtual cathode, is still ongoing. We will consider a scheme of injecting only sufficient electron charge to reduce the field to zero at the wall; such a scheme is used in microwave beam models for primary electron emission due to strong field effects at cold cathodes. 


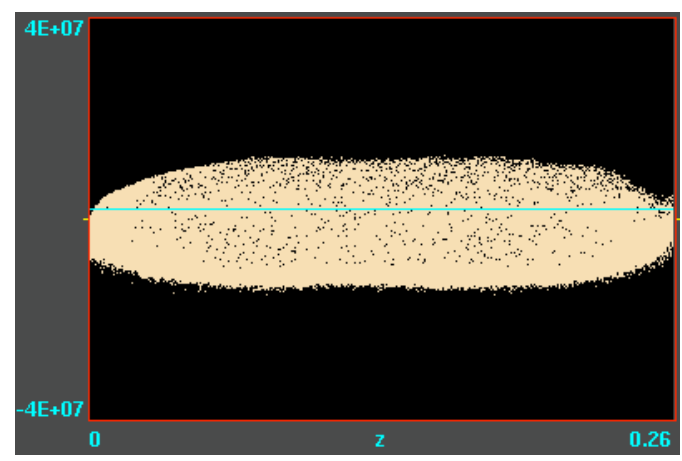

FIG. 25: Axial velocity phase space for the high resolution case at $2 \mu$ sec. Abscissa is $z(\mathrm{~m})$, ordinate is $v_{z}(\mathrm{~m} / \mathrm{s})$.

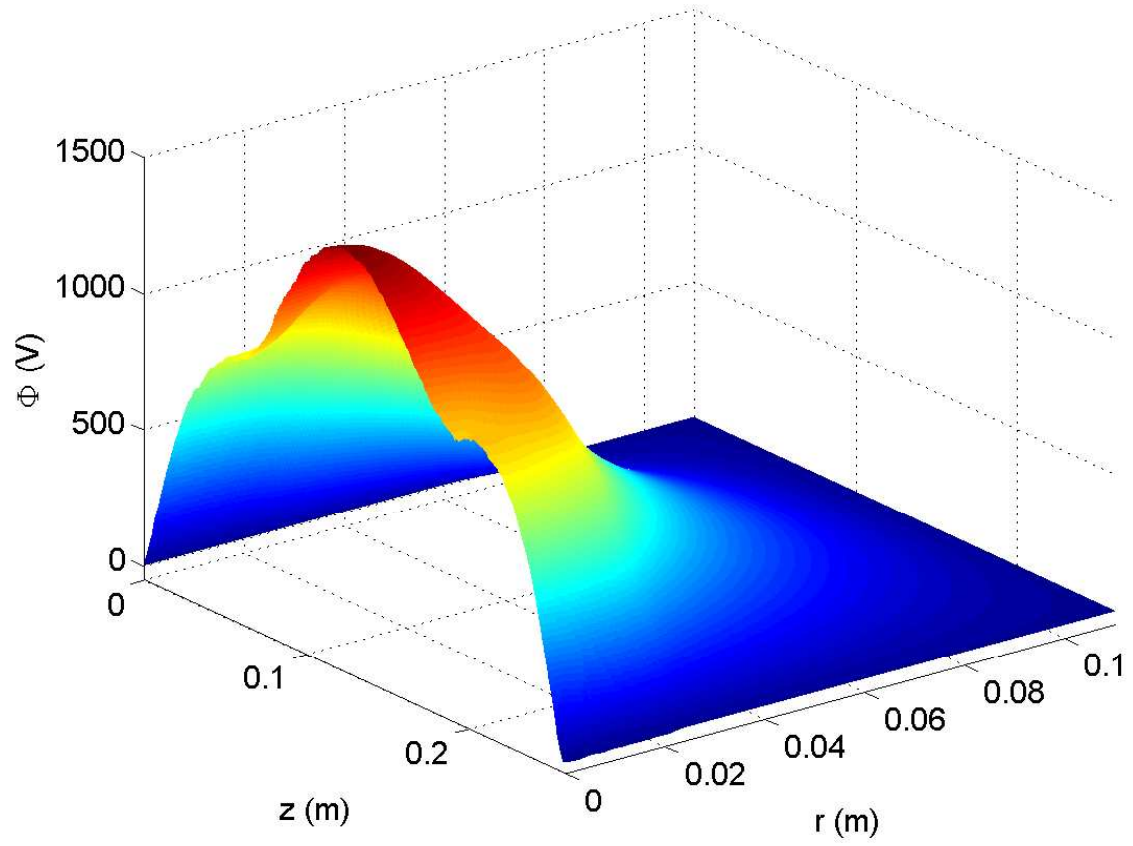

FIG. 26: Potential profile at $t=0.2 \mu$ sec.

\section{FUTURE PLANS}

There are a number of action items planned for the immediate future to improve our simulation capability. These include: (1) a proper model of the electron emission at the end wall, based on what we are learning from the detailed end-region simulations; (2) Full connection of the secondary-electron models from POSINST, in order to model secondaries 


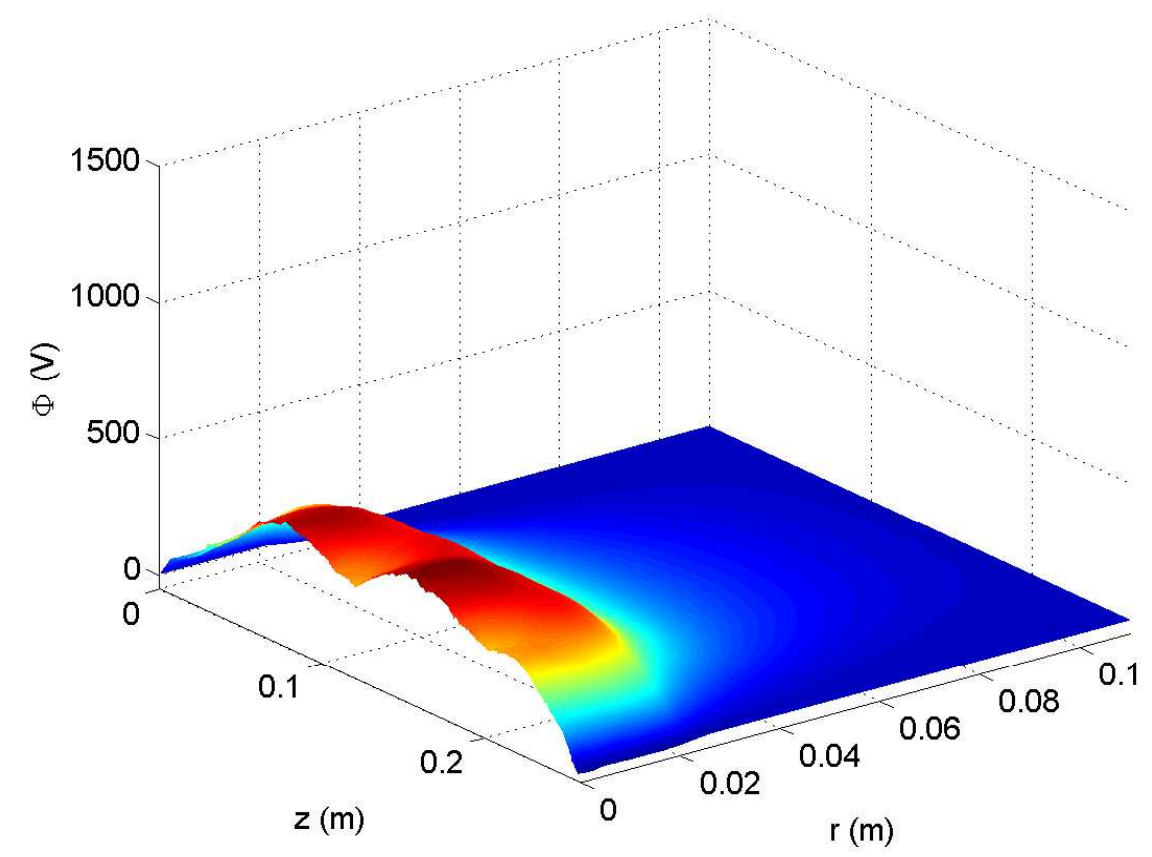

FIG. 27: Potential profile at $t=2 \mu$ sec.

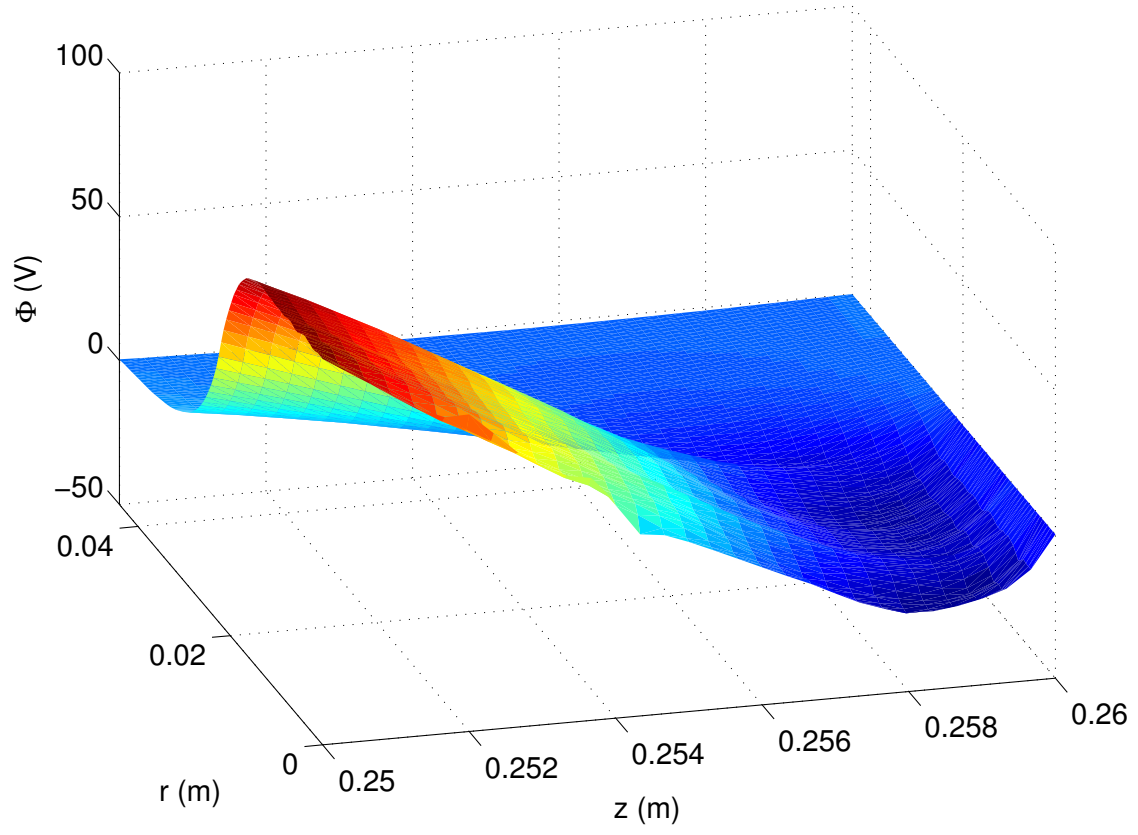

FIG. 28: Potential profile near the virtual cathode. The minimum occurs at about $-24 \mathrm{~V}$ about $1.67 \mathrm{~mm}$ from the end wall. 


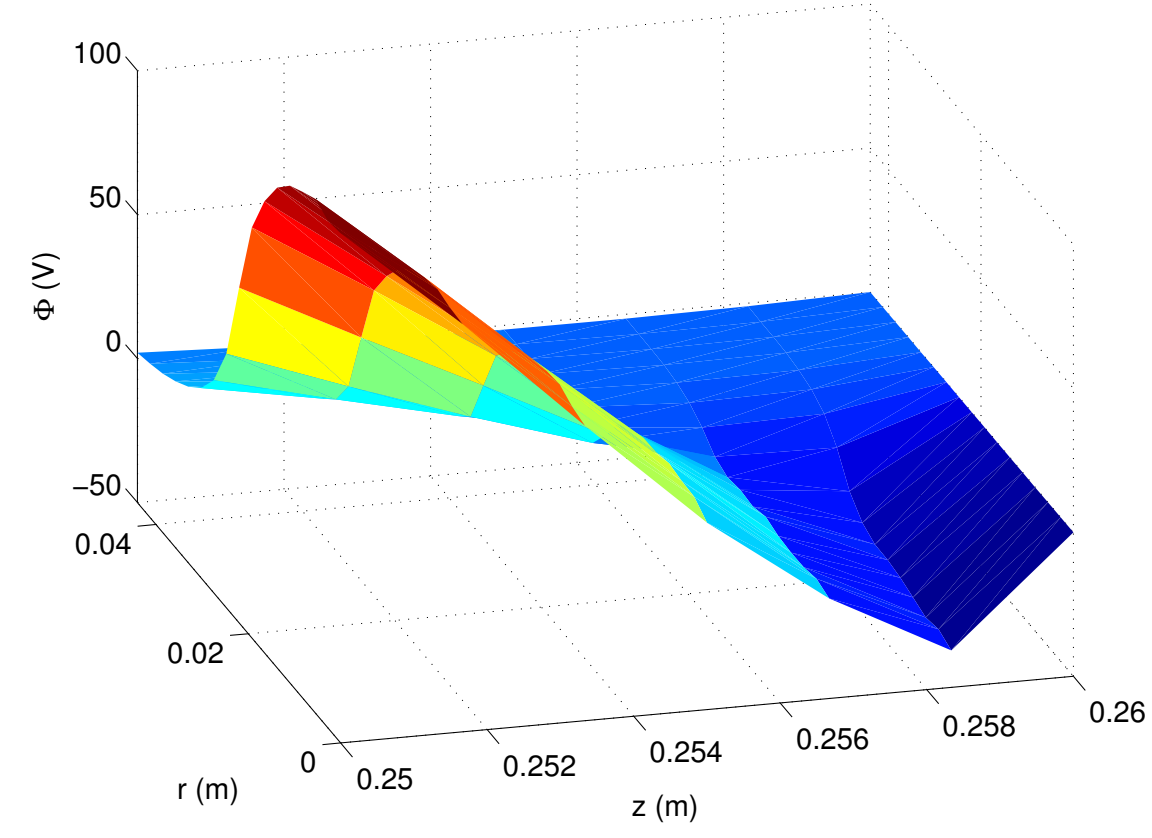

FIG. 29: Potential profile near the virtual cathode for the low resolution simulation. The minimum is about $-40 \mathrm{~V}$ at $2 \mathrm{~mm}$ from the end wall (the first cell).

released when electrons strike the beam pipe; (3) Simulations which take, as input, the ion beam distribution that is calculated to emerge from the electrostatic focusing section of HCX. We will undertake new 3-D two-species simulations with these capabilities and compare with experimental results.

We will also perform timestep-variation studies to assess the limits of validity of the interpolating electron mover. We have very limited data at the present time.

Over the next year we expect to complete the installation of the gas, reflected ion, and ionization modules and begin full-physics simulations of the programmatically more interesting case of electrons resulting from ion scrape-off at the beam pipe (with electrons resulting from direct desorption and from ionization of desorbed gas). Initially we will study situations with deliberately enhanced scrape-off, such that effects could be observed on experimental facilities as short as HCX. At some level these simulations can be done on workstations.

We also expect the code to be fully operational on NERSC's Seaborg and other parallelcomputing platforms. This entails resolving load-balancing issues that are complicated by the presence of the electrons. With a fully functioning parallelized code, we will be able to explore the more long-term issues of electron cloud effects from the levels expected through 
normal levels of ion scrape-off, requiring excellent statistics.

\section{Acknowledgments}

We thank D. P. Grote for invaluable assistance with the WARP code, and M. A. Furman for useful discussions. This work was performed under the auspices of the U.S. Department of Energy by Lawrence Livermore National Laboratory under contract W7405-ENG-48 and by Lawrence Berkeley National Laboratory under contract DE-AC03-76F00098, and under an SBIR grant at Tech-X.

[1] R. H. Cohen, A. Friedman, S. M. Lund, T. Azevedo, J.-L. Vay, "Electron-Cloud Effects on Heavy-Ion Beams.” DOE Milestone Report, March 2004.

[2] See, e.g., ECLOUD 04 Workshop proceedings, http://icfa-ecloud04.web.cern.ch/icfaecloud04/agenda.html

[3] L. Prost, et al., submitted to Phys. Rev. Special Topics-Accelerators and Beams (2004).

[4] P. H. Stoltz, M. A. Furman, J.-L. Vay, A. W. Molvik, and R. H. Cohen Phys. Rev. Special Topics-Accelerators and Beams 6, 054701 (2003).

[5] A. W. Molvik, M. Kireeff Covo, F. M. Bieniosek, L. Prost, P. A. Seidl, D. Baca, A. Coorey, and A. Sakumi, Phys. Rev. Special Topics-Accelerators and Beams 7, 093202 (2004); results extrapolated to normal incidence yielding an electron emission coefficient of six.

[6] R. H. Cohen, A. Friedman, S. M. Lund, A. W. Molvik, E. P. Lee, T. Azevedo, J.-L. Vay, P. Stoltz, S. Veitzer, "Electron-Cloud Simulation and Theory for High-Current Heavy-Ion Beams," Submitted to Phys. Rev. Special Topics-Accelerators and Beams, August 2004.

[7] S. E. Parker and C. K. Birdsall, "Numerical Error in Electron Orbits with Large $\omega_{c e} \Delta t, " J$. Comp. Phys 97, 91 (1991).

[8] See, e.g., R. H. Cohen, G. Rowlands, J. H. Foote, "Nonadiabaticity in Mirror Machines," Phys. Fluids 21, 14 (1978), and references therein.

[9] J. P. Verboncoeur, A. B. Langdon and N. T. Gladd, "An Object-Oriented Electromagnetic PIC Code", Computer Physics Communications 87, 199-207 (1995). 